\title{
Heterogeneous Effects of Intergenerational Social Mobility: An Improved Method and New Evidence*
}

\author{
Liying Luo \\ Department of Sociology \& Criminology \\ Population Research Institute \\ The Pennsylvania State University
}

Version: August 2021

\begin{abstract}
Citation: Luo, Liying. Forthcoming. "Heterogenous Effects of Intergenerational Mobility: An Improved Method and New Evidence." American Sociological Review

R package for implementing the method: MCM

Stata command for implementing the method: MCM_STATA
\end{abstract}

Key words: mobility effects models; intergenerational mobility; mobility-fertility hypothesis; interaction effects; diagonal reference model

*The study benefited from support provided by the Population Research Institute (NICHD P2CHD041025) and the Social Science Research Institute, the Pennsylvania State University. I thank Kira England, Andrew Halpern-Manners, James Hodges, John Iceland, Molly Martin, Susan McHale, Janet Novack, Léa Pessin, Michael Sobel, Jennifer Van Hook, and John Robert Warren for their helpful comments. I would also like to thank the ASR editors and the four anonymous reviewers, whose comments greatly improved the research. Earlier versions of this article were presented at the RC 28 Spring Meeting (March 2019, Frankfurt, Germany) and the annual meetings of the Population Association of America (April 2019, Austin). Please direct correspondence to Liying Luo, 202 Oswald Tower, University Park, PA 16802 or email liyingluo@psu.edu. 


\begin{abstract}
Intergenerational social mobility has immense implications for individuals' well-being, attitudes, and behaviors. However, previous methods may be unreliable for estimating heterogeneous mobility effects, especially in the presence of moderate- or large-scale intergenerational mobility. We propose an improved method, called the "mobility contrast model" (MCM). Using simulation evidence, we demonstrated that the MCM is more flexible and reliable for estimating and testing heterogeneous mobility effects, and the results are robust to the scale of intergenerational mobility. We revisited the debate about the effect of mobility on fertility and analyzed data from the 1962 Occupational Changes in a Generation Study (OCG-1) and more recent data from the 1974 through 2018 General Social Survey (GSS) using both previous models and the MCM. The MCM suggested a small association between fertility and occupational mobility in the GSS data but substantial and heterogeneous educational mobility effects on fertility in the OCG-1 and the GSS. Such effects were difficult to pinpoint using previous methods because mobility effects of different magnitudes and opposite directions among mobility groups may cancel out. The new method can be extended to investigate the effect of intergenerational mobility across multiple generations and other research areas including immigrant assimilation and heterogamy.
\end{abstract}




\section{Introduction}

Social scientists have long been interested in understanding the implications of intergenerational social mobility_ often conceptualized and measured as the difference between parents' (origin status) and adult children's socioeconomic position (destination status) — for individuals' attitudes, behaviors, and well-being outcomes (Akee, Jones, and Porter 2019; Chetty et al. 2014; DiPrete 2002; Friedman 2014; Iveson and Deary 2017; Yaish 2002). The mobility-fertility hypothesis is a prominent example: Researchers have hypothesized that because socially mobile individuals may experience loss of social network and difficulty in acculturation, they may lack social, emotional, and economic resources to support as many children as the socially nonmobile (Billingsley, Drefahl, and Ghilagaber 2018; Blau and Duncan 1967; Boyd 1994; Easterlin 1969, 1976; Goldscheider and Uhlenberg 1969; Lundberg 1991). However, empirical evidence about mobility effects $^{1}$ on fertility has been inconsistent with this hypothesis: Most studies have found that neither upward nor downward social mobility was related to number of children after considering origin and destination status (Blau and Duncan 1967; Sobel 1985; Stevens 1981; Tien 1961; Westoff et al. 1961; Zimmer 1981). Such null findings discouraged further investigations about mobility effects not only on fertility but also on other outcomes. It is unfortunate that this line of mobility research has subsequently remained relatively dormant despite the scientific and translational importance of intergenerational mobility (Billingsley et al. 2018; Zuanna 2007).

We contend that this puzzle may be due in part to the field's lack of an effective method for revealing the potential heterogeneity within status groups and between mobility groups. To be clear, a null finding does not equate to a faulty method, but until a key methodological problem is settled it is premature to abandon this important line of research. A standard regression approach to simultaneously estimating the effects of origin, destination, and mobility is problematic because 
the three variables are exactly related; that is, mobility is completely determined by destination and origin (Billingsley et al. 2018; Brody and McRae 1987; van der Waal, Daenekindt, and de Koster 2017). The field's state-of-the-art method for circumventing this challenge is the "diagonal reference model" (DRM) proposed by Sobel $(1981,1985)$. While the DRM is a rigorous and parsimonious method for studying mobility effects, the DRM's accuracy and reliability are unclear when mobility effects are heterogeneous among mobility groups (e.g., upward and downward mobilities have opposite effects) or when the scale of intergenerational social mobility is not small (e.g., the number of socially nonmobile individuals is smaller than the mobile).

We show that heterogeneity in mobility effects and mobility scale have critical implications for effects estimates in the DRM. As we will demonstrate in a simulation study, the DRM may yield inaccurate estimates of mobility effects when the scale of intergenerational mobility is nontrivial and mobility effects are heterogeneous. Our empirical investigation of the mobility-fertility hypothesis suggests that the null finding of mobility effects on fertility in the 1980s' debate may be at least in part attributed to heterogeneity in mobility effects on fertility; that is, mobility effects that are opposite in direction among mobility groups seem to cancel each other.

We propose a new method, called the "mobility contrast model" (MCM), for estimating and testing the effects of intergenerational social mobility. The MCM is more accurate than previous methods for identifying and estimating heterogeneous effects of intergenerational social mobility, and the results are robust to the scale of social mobility. Such mobility effects of different directions and magnitudes may have canceled out in previous studies, missing an opportunity to elucidate an important pathway through which socioeconomic positions may affect individuals' demographic, health, and other outcomes. This methodological improvement may facilitate 
renewed interest and developments in understanding the relationship between intergenerational mobility and a range of demographic, social, and well-being outcomes.

Our research contributes to the sociological literature in important ways. First, we propose an effective and reliable model for studying the effects of intergenerational social mobility on various outcomes. The R package MCM is designed to implement the new method. To help researchers make informed decisions in choosing a suitable method, we provide a best-practice guideline that describes the conditions under which one method may be preferred to others. Second, the methodological development proposed in this study is not only useful for stratification and mobility research but also other fields of sociological inquiry with similar methodological challenges. We discuss how the MCM can be extended to address important sociological research questions about immigrant assimilation and educational homogamy (as just two examples).

This paper proceeds as follows. We begin with a description of the methodological challenge in the classical mobility model, followed by a discussion of the limitations of previous methods that have been proposed to investigate mobility effects (Section 2). Next, we introduce the new MCM method, provide theoretical and methodological justifications for model specification and identification (Section 3), explain the similarities and improvement of the MCM compared to other methods, and demonstrate the advantages of the MCM with simulated data (Section 4). Sections 3 and 4 are fairly technical so some readers may prefer to skip them on a first read and proceed directly to the empirical example in Section 5, in which we reexamine the mobility-fertility hypothesis and compare model results from analyses of classical data from the Occupational Changes in a Generation Study of 1962 and more recent data from the 1974-2018 General Social Survey. We conclude by discussing the implications of the present study for related research areas and directions for future research. 


\section{Background}

Intergenerational social mobility occurs when adult children's socioeconomic position such as educational attainment, occupation, or income level is different from that of their parents. Intergenerational social mobility, including its magnitude, trends, and consequences, has been a classic topic in sociology, economics, and elsewhere (Aaronson and Mazumder 2008; Chetty et al. 2014; Hauser et al. 2000; Hout 1988; Long and Ferrie 2013; Song and Mare 2015; Xie 1992). At the societal level, intergenerational mobility has immense implications for social structures and inequality (Lillard and Willis 1994; Matras 1961; Simpson 1970; Stevens 1981; Torche 2015). At the individual level, mobility appears to affect attitudes, behaviors, and health outcomes. There are extensive literatures that examine associations between intergenerational mobility and a range of behaviors, attitudes, and outcomes including mental health conditions (Fox 1990; Houle and Martin 2011; Kessin 1971), health and well-being (Ahlburg 1998; Power, Matthews, and Manor 1996; Schuck and Steiber 2017), political attitudes and voting behaviors (Clifford and Heath 1993; Tolsma, de Graaf, and Quillian 2009; Weakliem 1992; Zang and Dirk de Graaf 2016), and vital rates (Billingsley et al. 2018; Blane, Harding, and Rosato 1999; Claussen et al. 2005; Kasarda, Billy, and West 1986; Tien 1961, 1967).

Whereas the effects of intergenerational social mobility on some domains are less debated (see, e.g., Knoke 1973; Nieuwbeerta, de Graaf, and Ultee 2000; Tolsma et al. 2009), research on its implication for fertility has been inconclusive. On the one hand, this topic has gathered a great deal of theoretical discussion. For example, the dissociative hypothesis (Blau and Duncan 1967; Nieuwbeerta et al. 2000; Sorokin 1927) predicts lower fertility among socially mobile couples on the basis that the acculturation stress and isolation that they may have experienced during social dislocation may interfere with their fertility desire, behaviors, and outcomes. The relative income 
theory (Easterlin 1969) also anticipates lower fertility for the downwardly mobile because they have limited resources relative to their tastes and desires for consumption preference formed in their origin family, and accordingly predicts higher fertility among the upwardly mobile.

On the other hand, despite this theoretical promise, empirical evidence about the mobilityfertility relationship has largely been inconsistent with what researchers have hypothesized. For example, in a series of studies, Westoff and colleagues $(1953,1961)$ compared fertility measures between the socially mobile and the nonmobile and found little evidence supporting the hypothesis that fertility is related to intergenerational occupational mobility. Later research in the 1970 s and 1980s also showed a general lack of mobility effects on the number of live births beyond an additive influence of origin and destination status (Duncan 1966; Jackson and Curtis 1972; Sobel 1985; Tien 1961, 1967; Zimmer 1981). This incongruence between theory and empirical evidence has served to diminish enthusiasm for pursuing further empirical investigations and the mobilityfertility inquiry in sociology has subsequently become relatively dormant.

However, until a critical methodological problem is settled, it may be premature to conclude that the mobility-fertility hypothesis should be discredited. That is, the unanticipated null results may be due to problems in modeling and estimating mobility effects, and perhaps researchers would have seen significant mobility-fertility association with a more effective method. We briefly describe below the methodological problem in the traditional mobility effect model and review two previous methods (including the current state-of-the-art method) to contextualize the motivation and specification of the new method that we propose.

To assess the effects of intergenerational social mobility, an intuitive starting point might be to compare the mean difference in the outcome (e.g., number of children) between the socially mobile and the nonmobile (see, e.g., Chaparro and Koupil 2014; James et al. 2006). The problem 
with this simple comparison is that the mobile and nonmobile groups not only differ in whether they experience social mobility but also their destination statuses. Consequently, any observed difference may or may not be attributed to mobility.

Lenski (1964) and Blalock (1967) were among the first to formally specify an intergenerational mobility effects model. Their model can be expressed using the following analysis of variance (ANOVA) model:

$$
g\left(E\left(Y_{i j}\right)\right)=\mu+\alpha_{i}+\beta_{j}+\gamma_{k},
$$

for origin status groups $i=1,2, \ldots, I$, destination status groups $j=1,2, \ldots, J$, and mobility group $k=1,2, \ldots, K . E\left(Y_{i j}\right)$ denotes the expected value of the outcome $Y$ for the $i$ th origin in the $j$ th destination; $g$ is the link function; $\alpha_{i}$ denotes the mean difference from the global mean $\mu$ associated with the $i$ th origin status category; $\beta_{j}$ denotes the mean difference from $\mu$ associated with the $j$ th destination status category; $\gamma_{k}$ denotes the deviation associated with the $k$ th mobility category. Lastly, a coding scheme is required to identify any ANOVA model like Eq. (1). ${ }^{2}$

However, the three predictors in Eq. (1) are linearly dependent: mobility = destination status - origin status. In other words, any two of the three variables completely determine the value of the third. The consequence of this linear dependence is that the model does not have a unique estimate even with the usual ANOVA coding. ${ }^{3}$ Several approaches have been developed to address this identification issue in mobility models, most notably Duncan' (1966) "square additive model" (SAM) and Sobel's $(1981,1985)$ "diagonal reference model" (DRM). ${ }^{4}$ We describe these two approaches below and discuss their strengths and limitations.

\subsection{Square Additive Model}

To address the identification problem, Duncan (1966) suggested modeling mobility as the interaction between origin and destination, a method that was later labeled the "square additive 
model" or SAM. That is, instead of including mobility as an independent and additive variable as in Eq. (1), Duncan considered mobility as the interaction term between the origin and destination main effects. As a result, the additive and independent quantity $\gamma_{k}$ is replaced with an interaction term $\delta_{i j}$ between origin status $\alpha_{i}$ and destination $\beta_{i}$. Duncan justified his model specification on the basis that one should not expect mobility effects when the patterns and variations in the outcome for the socially mobile can be adequately summarized or explained as an additive combination of the origin and destination status (1966: 93).

The upper-left panel in Table $1^{5}$ illustrates the SAM's parameterization of the status main effects, where $\alpha_{i}$ and $\beta_{j}$ are the same as defined in Eq. (1). The lower-left panel illustrates that the SAM uses $\delta_{i j}$-the interaction term of the $i$ th origin and $j$ th destination main effects corresponding to the $i j$ th mobility group - to model mobility effects. That is, for each origindestination combination, the expected value of the outcome $\mathrm{Y}$ can be expressed as the sum of the main effects $\alpha_{i}$ and $\beta_{i}$ and the mobility effect $\delta_{i j}$. For example, the expected value of the outcome for the two mobile groups moving from origin 1 to destinations 2 and 3 are $\alpha_{1}+\beta_{2}+\delta_{12}$ and $\alpha_{1}+\beta_{3}+\delta_{13}$, respectively.

[Table 1 about here]

A critical question is whether the interaction term $\delta_{i j}$ represents the sociological concept of a mobility effect. Statistically, $\delta_{i j}$ can be interpreted as the deviation associated with the $i j$ th (mobile or nonmobile) group from the expected value determined by the main effects $\alpha_{i}$ and $\beta_{i}$. However, such statistical quantities seem to depart from the conceptual idea of a mobility effect: whereas $\delta_{i j}$ represents the deviation from the two status main effects, a mobility effect is defined as the difference between the mobile and the nonmobile that can be uniquely attributed to mobilityrelated experience or characteristics. 
Scholars also argued that the SAM does not estimate the kind of status effect that sociologists have theorized. Specifically, Hope (1975) believed that the main effects of each class or status should be restricted to individuals who have lived that class "for life" (1975:336) and argued that the estimate of each class in Duncan's model was "contaminated" because it blends characteristics of the nonmobile and mobile individuals from different origins. Hope thus concluded that Duncan's model fails to estimate what the model was purported to test.

\subsection{Diagonal Reference Model}

Consistent with Hope's idea about status effects, Sobel (1981) developed the "diagonal reference model" or DRM that excludes the mobile and relies on the nonmobile for estimating status effects. That is, whereas six (and thus four freely-varying) parameters are used to represent each status of origin or destination in the SAM, the DRM focuses on the three status effects that lie on the upper-left-to-lower-right diagonal in the upper-middle panel of Table 1 , denoted by $\theta_{1}$, $\theta_{2}$, and $\theta_{3}$, respectively. Assuming no mobility effect, the means in the off-diagonal cells in the middle-upper panel of Table 1 can be expressed as a combination of the corresponding diagonal parameters $\theta_{i}$ and $\theta_{j}$ and a proportionality or salience parameter $\rho$. That is, a function of $\theta_{i}, \theta_{j}$, and $\rho$ is used to establish a baseline or referent to which the mobile may be compared for estimating the effect attributable to social mobility. For example, the hypothetical referent for the group moving from origin 1 to destination 2 is $\rho \theta_{1}+(1-\rho) \theta_{2}$, and for the group moving down from origin 3 to destination $1 \rho \theta_{3}+(1-\rho) \theta_{1}$. In a modified version (Sobel 1985), $\rho$ 's may vary across categories of origin status (or destination, but not both) and the interpretations are similar. For example, for those two mobility groups, the referents in the modified DRMs are $\rho_{1} \theta_{1}+(1-$ $\left.\rho_{1}\right) \theta_{2}$ and $\rho_{3} \theta_{3}+\left(1-\rho_{3}\right) \theta_{1}$, respectively, using origin-specific $\rho$ 's. 
Although the proportionality parameter $\rho$ plays a critical role in estimating the DRM and the $\rho$ estimate is available in a standard model output (e.g., the R package gnm), it is not a direct estimate of mobility effects. Rather, it is often interpreted as a salience parameter, indicating the relative importance of the origin and destination status effects.

To estimate mobility effects, the DRM adds a mobility variable or construct to the equation; for example, a dummy variable indicating mobility or nonmobility status or a categorical variable indicating upward mobility, downward mobility, or nonmobility. Such mobility variables represent different aspects of social mobility and standard regression interpretations follow straightforwardly. The lower-middle panel of Table 1 illustrates the parameterization of a DRM with upward and downward mobility indicators: the upward and downward mobility effects are modeled using two parameters $\tau_{1}$ and $\tau_{2}$ for the upper/right and lower/left off-diagonal cells, respectively. For example, for the two mobile groups moving from origin 1 to destinations 2 and 3 , the expected value of the outcome are $\rho \theta_{1}+(1-\rho) \theta_{2}+\tau_{1}$ and $\rho \theta_{1}+(1-\rho) \theta_{3}+\tau_{1}$, respectively.

It is important to note that although it may be theoretically interesting and desirable to simultaneously examine all types of mobility variables (i.e., mobility status, direction, and steps) in one equation, it has rarely been done in practice and only one of the mobility variables is typically included per equation. ${ }^{6}$ This is often because the statistical software would either give an error message or indicate "NA" in the output when more than one mobility variable is included. Methodologically, this means that the model is not identified, i.e., there are too many parameters for the data to distinguish. As a result, including two or more types of mobility variables in one DRM is often not a viable option. 
Nevertheless, with status and mobility parameters that correspond to meaningful sociological concepts, the DRM currently represents the most rigorous methodological approach and has been considered the state-of-the-art method in intergenerational mobility research. It has been widely used to study mental health (Houle 2011; Houle and Martin 2011), attitudes (Tolsma et al. 2009), political outcomes (Clifford and Heath 1993; Nieuwbeerta et al. 2000), and health conditions (van der Waal et al. 2017).

However, three questions remain unanswered about the DRM. First, the choice of excluding "newcomers" in estimating origin or destination status effects is justifiable when social mobility occurs at a relatively small scale. However, it may become problematic when social mobility is large in magnitude so that a significant number of members in one status consists of individuals with a different origin. For example, $65.9 \%$ of college-educated respondents in the 1976-2018 General Social Survey data were born to parents with a high school degree or less education. Although the nonmobile may still guard typical attitudes and behaviors of that social status group, their views and behaviors may also be influenced by the large number of the mobile from different socioeconomic backgrounds. In such scenarios, status effect estimates that rely solely on the nonmobile may be inaccurate.

Second, although Hendrikx and associates (1993) offered a mathematical exposition about the algorithm for computing the DRM's proportionality parameter $\rho$, its meaning and implication for estimating mobility effects have not been well understood. How is a mobility effect estimate based on $\theta$ 's and $\rho$ in the DRM different from or similar to an estimate based on main effects $\alpha$ 's and $\beta$ 's in the SAM? Section 4.2 discusses this question in detail.

Third, although mobility variables such as mobility status (socially mobile or nonmobile), step (number of social status levels that a person moves), and direction (upward or downward 
mobility) may concisely represent the association between mobility experience and the outcome of interest, such conciseness may come at the price of obscuring heterogeneity among mobility groups. ${ }^{7}$ That is, even with unique $\rho$ 's for each origin or destination level, DRM users necessarily assume that (1) a single proportion is adequate for all mobility groups from the same origin (or destination) status; and (2) mobility effects are homogeneous for all groups within a mobility category (e.g., for all upwardly mobile group or for all 1-step mobile groups) regardless of their origin or destination status. These simplifying assumptions may not be realistic when there is a large amount of heterogeneity among mobility groups. For example, when the effects of social mobility are opposite depending on their origin and/or destination, the DRM may incorrectly conclude that there exist no mobility effects when in fact important mobility effects of opposite directions cancel out.

\section{The Mobility Contrast Model: An Improved Method}

We propose a new method, called "the mobility contrast model" (MCM). The MCM extends the DRM in two ways: First, it is more flexible for estimating and testing the likely heterogeneous effects of intergenerational mobility. That is, if the mobility effect is in fact similar among mobility groups, the MCM and the DRM should yield similar estimates for mobility effects, and the DRM is preferred because of its parsimony. If, however, there exists substantial heterogeneity in mobility effects among mobility groups (i.e., different mobility groups have different effect sizes and/or opposite directions), the MCM makes it possible to estimate such distinct effects and thus should be helpful for identifying such distinct effects that may have been concealed using the DRM. Second, the MCM is more robust than the DRM to large-scale intergeneration mobility. In contrast, DRM estimates of mobility effects are sensitive to (i.e., differ depending on) the scale of mobility. 
As we will demonstrate in Section 4, the DRM estimates differ from the data-generating parameters when mobility effects are heterogeneous and/or the scale of intergenerational mobility is moderate or large, a situation for which the DRM is not suitable.

We describe below how the new model is specified, followed by theoretical motivations and methodological justifications. Given the long-standing identification problem in mobility effect models, we also explain how the MCM is identified with different coding schemes (e.g., dummy coding of an education variable, which sets high school graduates as the reference group or effect coding in which the effects of all education groups sum to zero). The R package MCM can be downloaded from CRAN to implement the method.

The mobility contrast model (MCM) consists of two steps. Whereas Step 1 estimates status main effects and is thus identical to the SAM, Step 2 estimates mobility effects as a set of originstatus interaction contrasts, reflecting the conceptual and methodological distinction of the MCM from the SAM proposed by Duncan (1966).

Step 1. Fit a generalized linear model that includes the origin main effects, destination main effects, and their interaction terms:

$$
g\left(E\left(Y_{i j}\right)\right)=\mu+\alpha_{i}+\beta_{j}+\delta_{i j}+\sum_{1}^{C} \eta_{c} x_{c},
$$

where $E\left(Y_{i j}\right), g, \alpha_{i}, \beta_{j}$, and $\delta_{i j}$ are the same as defined earlier, and the usual ANOVA coding (i.e., effect or sum-to-zero coding) applies to each effect; and $\eta_{c}$ denotes the coefficient for the $c$ th covariate $x_{c}$ such as age, sex, or race.

Step 2. Create a set of origin-specific contrasts of the interaction terms $\delta_{i j}$ to estimate mobility effects. Specifically, as Table 1's lower-right panel illustrates, the MCM uses $\delta_{i j}-\delta_{i i}$ to represent the mobility effect with status main effects purged, where $\delta_{i j}$ is the deviation from the main effects $\alpha_{i}$ and $\beta_{j}$ associated with the mobility group from origin $i$ to destination $j$, and $\delta_{i i}$ is 
the deviation from the main effects $\alpha_{i}$ and $\beta_{i}$ for the nonmobile of the origin $i$ (and thus destination $i$ ). For example, in the lower-right panel of Table 1 the mobility effect for the group who rose from the most disadvantaged background (i.e., origin status 1) to the highest status in adulthood (destination status 3 ) is modeled using the difference between $\delta_{13}$ and $\delta_{11}$, which correspond to the interaction term of the two-step upward mobility group and the nonmobile group from the same origin, respectively.

\subsection{Status Effects in the MCM}

In the following we describe the theoretical motivation for the statistical specification of the status effect and mobility effect in the MCM. As Table 1's upper panel shows, unlike the parameterization of the DRM, which focuses on typical characteristics of a status, denoted by the $\theta$ 's, the MCM and the SAM focus on the main effects of origin or destination status denoted by the $\alpha$ 's and $\beta$ 's, respectively. As discussed below, the two types of status effects are related but distinct sociological substances.

The MCM focuses on main effects for two reasons. First, while status effects are essential and meaningful quantities in sociological research, they are considered in the analysis of intergenerational mobility effects to reflect the distinct social norms and conditions that individuals are exposed to. From this exposure perspective, when estimating the effect of an origin status, it is reasonable to consider all individuals from that origin regardless of their mobility status (mobile or nonmobile) because both groups may be exposed to similar attitudes and behaviors in their formative ages. A fundamental premise of intergenerational mobility research is that such earlylife conditions may have long-lasting impacts on their behaviors and well-being. For similar reasons, it should be reasonable to include both the nonmobile and mobile of the same destination status to estimate the effect of destination status. 
Second, inclusion of the socially mobile in estimating main effects of the origin and destination status may be substantively important and meaningful in the presence of non-trivial social mobility. It is completely justifiable to focus on the nonmobile when mobility is relatively uncommon. However, as Sobel (1981:904) noted, when social mobility is not uncommon or occurs at a large scale, it becomes questionable to exclude the mobile from estimating the status effect. Also, including the socially mobile of the same origin or destination in estimating a status effect recognizes that the social interactions between social groups are not one-directional; that is, the mobile and the nonmobile may exert influences on each other.

\subsection{Mobility Effects in the MCM}

Although the MCM's status parameterization is the same as the SAM's for reasons elaborated above, its mobility effect parameters differ from the SAM's because, as critics have pointed out, the unstructured interaction term $\delta_{i j}$ in the SAM does not directly correspond to the sociological conceptualization of mobility effects. Specifically, under sum-to-zero coding, $\delta_{i j}$ represents the deviation associated with the $i j$ th mobility group from the two status main effects. In contrast, a mobility effect is conceptualized as the unique impact of the mobility experience or some distinct characteristics of the mobile group that cannot be attributed either to the origin or destination main effects. This discrepancy between the conceptualization and the operationalization of mobility effects is, we argue, the main limitation of the SAM.

Step 2 of the MCM addresses this concern by creating a set of contrasts in the interaction terms to directly estimate and test the difference between the mobile and the nonmobile after removing the status main effects. That is, the contrast approach considers an intergenerational mobility effect as a special structure or contrast of the interaction terms that is origin-specific, as Table 1's lower-right panel illustrates. For example, the mobility effect for the group who moved 
from origin status 3 to destination 2 is modeled as $\delta_{32}-\delta_{33}$, where $\delta_{32}$ is the interaction term of the one-step downward mobility group from origin 3 and $\delta_{33}$ is the interaction term for the nonmobile of origin 3. Intuitively, this idea can be understood as first removing the main effects of origin and destination status, and then directly comparing the deviations of the mobile and the nonmobile of the same origin. This step is a critical improvement from the SAM, where analysts rely on the unstructured $\delta_{i j}$ 's to examine mobility effects.

We emphasize that conceptually, the mobility effect parameters in the MCM (i.e., a set of origin-destination interaction contrast) and the SAM (i.e., unstructured interaction) are distinct conceptual quantities; we have argued above that the MCM's parameterization is more consistent with the sociological concept of intergenerational mobility effect than the SAM's. Statistically, the effect estimates and standard errors for the MCM's and the SAM's mobility parameters will naturally differ. As we will demonstrate using simulation and real-world empirical examples, the two methods can lead to largely different substantive conclusions about the magnitude and significance of mobility effects on an outcome.

\subsection{Model Identification, Interaction, and Coding Scheme}

Given the linear dependence in the traditional mobility effect models (1), it is important to emphasize that the MCM in Eq. (2) is identified and thus does not require additional constraints other than a coding scheme (e.g., dummy coding by setting high school graduates as the reference group, or effect coding, setting the effects of all education groups to sum to zero). This means that a unique set of parameter estimates for the MCM fits the data better than others. However, this does not imply that all of the $O \times D$ interaction parameters $\delta_{i j}$ 's vary freely. Like any fully identified ANOVA or generalized linear model, the interaction terms in the MCM have $(O-$ 1) $\times(D-1)$ degrees of freedom, and the remaining $O+D-1$ interaction terms are determined 
by the coding scheme. The qualitative difference between the unidentified model (1) and the MCM is that the latter requires no additional constraint to identify the model besides the usual coding scheme, whereas the unidentified model (1) does require at least one more constraint in addition to the usual coding scheme. The model is identified as any generalized linear model, and covariates such as age, sex, or race can be added as in a standard regression model.

Because the MCM characterizes mobility effects in terms of a structure of the interaction terms between origin and destination, it is critical to clarify the equivalence of coefficient estimates among different coding schemes (e.g., dummy coding or sum-to-zero coding); this means that the estimated design cell means (i.e., the estimated mean for each origin-destination combination) are invariant across coding schemes. Although this equivalence holds for both main effects and interaction estimates in the MCM, it is less obvious for interaction terms than for the origin and destination main effects. In fact, the estimated interaction terms (and main effects) under different coding schemes necessarily have different numerical values because their references differ. For example, under the effect coding (i.e., the sum-to-zero coding), the cell means of the origin 1destination 1 and origin 1-destination 2 combination are parameterized as $\alpha_{1}+\beta_{1}+\delta_{11}$ and $\alpha_{1}+$ $\beta_{2}+\delta_{12}$, where $\alpha_{1}, \beta_{1}$ and $\beta_{2}$ denote the deviations from the grand mean $\mu$ associated with origin 1 , destinations 1 and 2, respectively; $\delta_{11}$ represents the deviation associated with the nonmobility group from the sum of the main effects $\alpha_{1}+\beta_{1}$; and $\delta_{12}$ represents the deviation associated with the mobility group from the sum of the main effects $\alpha_{1}+\beta_{2}$. In contrast, under the dummy coding with origin 1 and destination 1 omitted as the reference group, that reference group's cell mean is treated as the intercept, denoted as $\mu^{\prime}$; the difference between the cells origin 1-destination 2 minus origin 1-destination 1 is the "main" effect of destination 2, denoted as $\beta_{2}^{\prime}$; the difference between the cells origin 2-destination 1 minus origin 1-destination 1 is the "main" effect of origin 2 , 
denoted as $\alpha_{2}^{\prime}$; and the cell mean of origin 2-destination 2 minus the cell means of origin 1destination 2 and origin 2-destination 1, plus the cell mean of origin 1 -destination 1 is the interaction effect, denoted as $\delta_{22}^{\prime}$. The numeric values of the estimated main and interaction effects

(i.e., $\hat{\alpha}_{2}, \hat{\beta}_{2}, \hat{\delta}_{22}, \hat{\alpha}_{2}^{\prime}, \hat{\beta}_{2}^{\prime}$, and $\hat{\delta}_{22}^{\prime}$ ), therefore, necessarily differ between the two coding schemes due to a shift in what these quantities represent. However, such differences do not arise from an identification problem, which qualitatively distinguishes the MCM from the traditional model (1).

We recommend effect or sum-to-zero coding for the MCM because the goal is to establish a common reference from which each origin-destination combination may deviate (Aiken, West, and Reno 1991; Jaccard and Turrisi 2003). That is, the effect coding is preferred for the MCM because the parameters all have the same referent group - the next lower level in the hierarchy of main effects and interactions. For example, for the origin and destination main effects, the same referent group at the lower level is just the grand mean $\mu$. For the interaction $\delta_{i j}$, the referent at the next lower level is the origin main effect $\alpha_{i}$ and destination main effect $\beta_{j}$. Such a common referent lays the foundation for Step 2 in the MCM, which estimates and tests a set of contrast in those interactions, and more importantly, facilitates interpretation of the estimates in a way that is consistent with the sociological conceptualization of mobility effects.

\subsection{Improvement and Strengths of the MCM}

In the preceding exposition, we have argued by specifying mobility effects as a set of origin-destination interaction contrasts, the MCM better represents the conceptualization of mobility effect than the SAM's unstructured interaction. Compared to the DRM, the MCM is particularly useful for detecting heterogeneous effects of intergenerational mobility, especially in the context of large-scale social mobility, although the DRM's parameterization of mobility effects is conceptually similar to that in the MCM (see a detailed exposition in Section 4). 
Methodologically, the MCM does not assume any functional form of the mobility effect in relation to the origin and destination and allows the mobility effect parameters to vary among mobility groups in a more flexible way than the DRM. For example, it allows the mobility effect for the group moving from origin 1 to destination 3 to differ from the group moving from origin 2 to destination 3. In contrast, potential heterogeneity may be concealed using mobility status indicators or difficult to pinpoint using mobility direction or step variables in the DRM.

We emphasize that conceptually, by modeling mobility effects as a set of interaction contrasts between the origin and destination status, the MCM represents the theoretical consideration that mobility effects should be considered in relation to the origin and destination status. Moving up or sliding down on the status ladder may have very different meanings and implications depending on the specific status of origin and destination. For example, a child born to parents with doctoral degrees whose own highest education level is college - that is, one-step downward mobility on the educational attainment hierarchy—may not experience any significant amount of change in life quality, stress, or social network disruption. In contrast, an individual born to high school educated parents who did not complete high school may experience a qualitative downgrade of living conditions and a considerable amount of stress. By modeling mobility effects as a set of contrasts in interaction terms between the origin and destination status, the MCM acknowledges the conceptual dependence between mobility and the statuses as Duncan (1979) advocated.

We also clarify that because the MCM specifies mobility effects as a set of origindestination interaction contrasts, the MCM has the same overall model fit statistics (e.g., the AIC statistic) and degrees of freedom (i.e., the number of freely-varying parameters) as the SAM. However, the MCM is not designed to improve such model fit statistics; rather, the primary goal 
of this method is to specify effect parameters that, conceptually, more accurately estimate the sociological process of interest-intergenerational mobility. That is, although the MCM and the SAM do not differ in overall model fit statistics, the two models' mobility effect parameters represent distinct conceptual and statistical quantities. As we will demonstrate using simulation and empirical examples, the effect estimates and standard errors for the MCM's and the SAM's mobility parameters will subsequently differ, leading to different substantive conclusions about the magnitude and significance of mobility effects on the outcome between the two models.

Because of conceptual limitation of the SAM's mobility effect parameters, in the section that follows, we focus on comparing results from the MCM and the DRM. Using four simulations that represent scenarios with varying degrees of heterogeneity in mobility effects and mobility scale, we show that the MCM is more useful when mobility effects differ in magnitude and direction among mobility groups, especially when intergenerational mobility is non-trivial. These substantively meaningful heterogeneities are otherwise difficult to detect or interpret using the DRM.

\section{Mobility Contrast Model and Diagonal Reference Model Compared: Simulation Evidence and Practical Guideline}

We have argued that conceptually, the mobility parameters in the MCM and DRM better correspond to the conceptualization of mobility than the SAM. Given the apparent difference in model specification of the MCM and the DRM, what model should one choose to investigate intergenerational mobility effects? Under what conditions do the MCM and the DRM give different conclusions? To help researchers make an informed choice of method, we compare and contrast the MCM and the DRM in scenarios with varying degrees of intergenerational mobility 
(i.e., the ratio of number of movers relative to stayers) and heterogeneity in mobility effects (i.e., the degree to which the directions and magnitudes of mobility effects vary among mobility groups).

\subsection{The Equivalence between the MCM and the DRM with Homogeneous Mobility Effects}

We begin with the simplest scenario in which the mobility effects are homogeneous, i.e., the effects are the same across all mobility groups. Under this condition, the DRM's mobility effect estimates are just (weighted) averages of the mobility effect estimates in the MCM, even in the presence of large-scale intergenerational mobility. To help readers understand this assertion, it is necessary to clarify two questions: (a) how the proportionality parameter $\rho$ is determined in the DRM and (b) how mobility effects are parameterized and estimated in the DRM and in the MCM.

Sobel (1981) used the maximum likelihood method to estimate the proportionality parameter $\rho$. It implies that, as Sobel carefully noted, the estimate of $\rho$ is "not solely a function of the diagonal sample means" (1981: 899, footnote 8) but also depends on off-diagonal means and the distribution of observations in the mobility table. Hendrickx and colleagues (1993: 342-343) provided further insight into the statistical implication of the proportionality parameter $\rho$ for estimating the mobility effect in the DRM. Specifically, these authors showed that assuming small-scale mobility, the origin-specific proportion parameter $\rho_{i}$ is in fact the ratio between the origin main effect and the sum of the origin and destination main effects $\rho_{i}=\frac{\alpha_{i}}{\alpha_{i}+\beta_{i}}$; the destination-specific proportion $\rho_{j}$ is the ratio between the destination main effect and the sum of the origin and destination main effects $\rho_{j}=\frac{\beta_{j}}{\alpha_{j}+\beta_{j}}$. This clarifies the relationship between the proportionality parameter $\rho$ and the status main effects parameters $\alpha_{i}$ and $\beta_{j}$.

This clarification about $\rho$ has an important implication for understanding the relationship between the mobility effect parameters in the DRM and the MCM. To illustrate, assume a best 
model-fit scenario in which each cell in a mobility table has two unique proportion parameters, one for the origin and the other for the destination. Then the referent for a group from origin $i$ and destination $j$ is $\rho_{i} \theta_{i}+\rho_{j} \theta_{j}$. For example, the referent for the group from origin 1 and destination 2 can be expressed as $\rho_{1} \theta_{1}+\rho_{2} \theta_{2}$. Replacing $\rho_{1}$ with $\frac{\alpha_{1}}{\alpha_{1}+\beta_{1}}, \theta_{1}$ with $\alpha_{1}+\beta_{1}, \rho_{2}$ with $\frac{\beta_{2}}{\alpha_{2}+\beta_{2}}$, and $\theta_{2}$ with $\alpha_{2}+\beta_{2}$ results in the referent being simply $\alpha_{1}+\beta_{2}$.

The above exposition suggests that despite the superficial difference, the choice of parameterizing status main effects or life-time status effects are statistically equivalent for representing cell means, at least in the best model-fit scenario. It means that, although the DRM and the MCM parameterize status effects differently (i.e., life-time status effects or status main effects), the different parameters are just different ways for describing status effects with similar abilities to account for data variation. To this extent, different parameterizations of the status effects in the DRM and the MCM are methodologically equivalent. ${ }^{8}$

The other seeming difference that obscures the relationship between the DRM and the MCM is how the two parameterize mobility effects. To quantify mobility effects, the MCM uses the difference between two interaction terms after removing the origin and destination main effects - that is, $\delta_{i j}-\delta_{i i}$-while the DRM uses mobility variables such as mobility status, direction, and step. However, it is important to note that both methods specify mobility effects as the difference between the socially mobile and the nonmobile after removing status effects. Also recall that the status effects are statistically equivalent between the MCM and the DRM in terms of the representation of the cell mean. It follows that for given data the variation that is not explained by status effects in each cell should also be the same. That is, the MCM and the DRM rely on the same quantities that are being expressed in different forms: the former models it as a 
specific structure of the interaction terms $\delta_{i j}{ }^{\prime}$ s, and the latter an average difference between the mobile and the nonmobile.

Specifically, including mobility variables such as mobility status, direction, and step in the DRM is equivalent to estimating the average deviations that remain after considering status effects between the socially mobile and the nonmobile. That is, the kind of mobility effects estimated in the DRM can be expressed as an averaged difference between the specific mobility group and the nonmobile of the same origin. Consider the example of Table 1 with three origin and destination categories. After estimating life-time status effects in the baseline model, the DRM models mobility status effect using a mobility status indicator, which has the effect of parameterizing the mobility status effect as $\frac{1}{6}\left(\left(\delta_{12}+\delta_{13}+\delta_{21}+\delta_{23}+\delta_{31}+\delta_{32}\right)-2 *\left(\delta_{11}+\delta_{22}+\delta_{33}\right)\right)$.

Similarly, the effect of upward mobility is equivalent to $\frac{1}{3}\left(\left(\delta_{12}+\delta_{13}+\delta_{23}\right)-\left(\delta_{11}+\delta_{11}+\right.\right.$ $\left.\delta_{22}\right)$ ). That is, the mobility effect in Sobel's model is in fact an average of the MCM's $\delta_{i j}-\delta_{i i}$, assuming that the DRM with fewer parameters fits the data equally well as the MCM with more parameters. It implies that when the mobility effects are truly homogeneous among mobility groups regardless of their origin or destination-i.e., when $\delta_{i j}-\delta_{i i}$ are the same for any origin $i$ and destination $j$-the mobility effect estimates in the DRM and the MCM should be the same.

\subsection{Heterogeneous Mobility Effects and Mobility Scale}

The equivalence between the DRM and the MCM holds on the condition that mobility effects are homogeneous across mobility groups. When the sizes and directions of the mobility effects vary among mobility groups, however, conclusions about mobility effects from the DRM may differ, sometimes substantially, from the MCM. This is because, as Sobel $(1981,1985)$ noted, the maximum likelihood estimator of the salience or weight parameter $\rho$ is a function of status main effects, mobility effects, and distribution of observations per origin-destination group $-\mathrm{a}$ 
distribution parameter that determines the scale of intergenerational social mobility. When mobility effects are truly homogeneous across mobility groups and the stayers represent the vast majority in each status, the DRM's salience parameter $\rho$ largely depends on status main effects, establishing the key condition for the equivalence between the DRM and the MCM. Different estimates of mobility effects between the MCM and the DRM should be expected when mobility effects differ in size and/or direction among mobility groups or stayers are no longer the vast majority in a socioeconomic status.

To demonstrate the implications of heterogeneity in mobility effects and mobility scale for the DRM and the MCM, we present simulation study that assesses the two models by comparing each method's estimates against the true effect parameters in the data-generating mechanism. We generate four particular examples with little sacrifice of generality: a design with $O=3$ origin statuses, $D=3$ destination statuses, and a normally distributed outcome. Each simulation represents one of the four scenarios, namely, homogeneous mobility effects with small-scale mobility, homogeneous effects with moderate-scale mobility, heterogeneous effects with smallscale mobility, and heterogeneous effects with moderate-scale mobility. Although it is straightforward to simulate data using larger mover-stayer ratio, say, 4:1 to approximate largescale mobility, the DRM often fails to converge, providing no coefficient estimates. We thus focus on small- and moderate-scale mobility for model comparison and demonstration purposes.

For homogeneous mobility effects, all off-diagonal parameters, i.e., mobility effects, are fixed at 0.4 . For heterogeneous effects, off-diagonal mobility effects parameters differ in size and direction. To simulate small- and moderate-scale mobility, we set the mover-stayer ratio to be at 1:4 and 2:3, respectively. The data are otherwise the same including total sample size $(\mathrm{N}=30,000$ observations in each data), the overall mean $(\mu=1.5)$, origin main effects $\left(\alpha_{i}=0.6,-0.2,-0.4\right)$, 
destination main effects $\left(\beta_{j}=1,-0.2,-0.8\right)$, and the error standard deviation $(\sigma=0.1)$. Table 2 presents mobility effect parameters and mobility scales used in each simulation. We provide the $\mathrm{R}$ code that we used to generate and analyze these datasets in the Appendix so readers can see for themselves and/or manipulate the simulation parameters.

[Table 2 about here]

For each simulation scenario, we fit three linear DRMs with a constant $\rho$ and one of the three mobility variables: a dummy variable indicating mobility status (nonmobile or mobile), a categorical variable indicating the direction of the mobility (nonmobile, downwardly mobile, or upwardly mobile), and a categorical variable indicating the number of steps that a hypothetical person moves on the socioeconomic ladder $(0,1$, or 2 steps $) .{ }^{9}$ The $\mathrm{R}$ package "gnm" was used for fitting DRMs. We fit one linear MCM that includes status main effects and a contrast of the originstatus interaction terms to each data. All models were fit by maximizing the likelihood.

Table 3 reports coefficient estimates for mobility variables in the DRM in four scenarios that vary in the degree of heterogeneity in mobility effects and mobility scale. Shaded cells indicate estimates that differ from the true parameters, which range from -0.2 to 1.0 in Table 2 , by 0.05 or more. As Table 3's left panel shows, the DRM often accurately estimates mobility effects ( $\widehat{\tau}_{l} \approx 0.4$ for most cases) when the effects are truly homogeneous $\left(\tau_{i}=0.4\right.$ for all $\left.i^{\prime} s\right)$, although the effect estimates for downward and upward mobility are slightly less accurate. The DRM estimates are somewhat less accurate with moderate-scale intergenerational mobility than smallscale mobility.

[Table 3 about here]

However, when mobility effects are in fact heterogeneous in magnitude and direction, as shown in the right panel in Table 3, the DRM estimates of mobility effects are less accurate, even 
with small amount of intergenerational mobility. For example, the DRM suggests a score that was -0.522 lower among the downwardly mobile, whereas the mean of the true effects of downward mobility — the data-generating parameters—is $0.267\left(\frac{-0.4+0.5+0.7}{3}\right)$. The DRM estimates of mobility steps ( 0.224 and 0.006 for steps 1 and 2, respectively, in Table 3's upper-right panel), however, are close to the true effects $\left(\frac{-0.4+0.5-0.2+1}{4}=0.225\right.$ and $\frac{-0.7+0.7}{2}=0$, respectively, as shown in Table 2).

The DRM estimates appear to be unreliable in the joint presence of moderate-scale intergenerational mobility and heterogeneous mobility effects, as reported in Table 3's right panel. For example, the upward mobility was estimated to have 1.091 higher score, although the true effect is much smaller $\left(\frac{-0.2-0.7+1}{3}=0.033\right)$. Even the previously accurate estimates of mobility steps ( 0.421 and 0.138 for 1-step and 2-step mobility, respectively) now depart from the "truth" (0.225 and 0 , respectively).

Table 4 presents mobility effect estimates from the MCM. The MCM yields accurate and reliable estimate of mobility effects and is robust to effect heterogeneity and mobility scales. For example, even in the most challenging scenario with moderate mobility and mobility effects of opposite directions and varying effect sizes, the MCM estimates of the two 2-step mobility (-0.700 for 2-step upward mobility group and 0.699 for 2-step downward mobility) are faithful to the datagenerating parameters (-0.7 and 0.7 , respectively).

[Table 4 about here]

The deviance statistics and the AIC suggest that the MCM fit the data better than the DRM across all four scenarios. The DRM fit the data best with homogeneous mobility effects and smallscale mobility and worst with heterogeneous effects and moderate-scale mobility. Such model fit evidence is consistent with the above comparison of the true parameters and the estimates. 
Our simulation demonstration and mathematical exposition shed light on model choice for studying mobility effects. We recommend the following best-practice guideline: When the investigator has theoretical reasons to expect homogeneous mobility effects (i.e., the effect size or direction does not vary across mobility groups) and empirical evidence that intergenerational social mobility occurs at a small scale (i.e., the nonmobile group largely outnumbers mobility groups combined), the DRM may be preferred as a parsimonious model that yields reliable estimates. However, when theory is lacking about the magnitude or direction of mobility, the MCM should be preferred to the DRM, especially when the distribution of a mobility table suggests moderate or large amount of intergenerational mobility. If the goal is to identify mobility groups that may have distinct outcomes or characteristics, the MCM serves that purpose better than the DRM.

\section{Mobility-Fertility Example}

This section revisits the mobility-fertility hypothesis by comparing the MCM and the DRM by analyzing the same data with the same fertility outcome variable and predictors as in the 1980s' debate as well as more recent data. Note that because the SAM's unstructured interaction terms do not represent mobility effects that researchers intend to estimate (Hope 1975; Sobel 1981, 1983), the results from the SAM cannot provide direct evidence about the mobility-fertility hypothesis. The following discussion thus focuses on comparing the MCM and the DRM results; Table S4 reports the results from the SAM. Here, we do not attempt to provide a full assessment of the mobility-fertility hypothesis; the main objective of this exercise is to demonstrate how the MCM can be used to address an important and sociologically interesting question by revealing the 
heterogeneity in mobility effects on fertility. Such substantively meaningful heterogeneities are difficult to detect or interpret using the DRM.

The dissociative hypothesis predicts both upward and downward mobility to be associated with lower fertility because mobility disrupts social network and family ties and thus implies less support for child rearing (Sorenson 1989; Sorokin 1927). The selection hypothesis contends that teen parenthood and a large number of children may limit education and occupation opportunities and consequently mobility likelihood (Van Bavel 2006), contributing to differential fertility among SES groups (i.e., higher fertility in low SES groups and lower fertility in high SES groups)(Chu and Koo 1990; Mare 1997). These competing forces and mechanisms may operate to cancel each other and result in similar fertility between the socially nonmobile and mobile, especially for individuals who moved to an adjacent status. However, prior literature suggested that mobility effects may be most evident among extreme mobility group who moved far away from their origin status (Freitas et al. n.d.; Friedman 2014; Southgate et al. 2017). Based on such theoretical accounts and empirical evidence, we thus expect upward mobility from the most disadvantaged family origin to the top status to have significantly lower fertility, and other mobility groups to have a similar fertility level as the nonmobile.

\subsection{Data and Measures}

We used the DRM and the MCM to analyze the data from the Occupational Changes in a Generation, 1962 (OCG-1) - the main dataset used in the 1980s' debate about modeling intergenerational mobility effects - and the 1974-2018 General Social Survey (GSS) data. Following Blau and Duncan (1967) and Sobel (1981), we restricted the OCG-1 analyses to USborn White men whose wives were 42-61 years old and living in the same household. For the GSS, we selected US-born White men aged 40-64 to facilitate model comparison. 
We excluded women from the empirical demonstration for two reasons. First, the purpose of the empirical analysis is to compare model results from prior studies and the MCM. Because for historical reasons prior research mostly focused on men, we followed the tradition of using data from male respondents in the GSS to maximize comparability. Second and more importantly, women's education, occupation, and labor force participation have changed dramatically in the US since the 1970s. Consequently, the implications of women's educational and occupational mobility for fertility may be very different from those for men. Although it is a highly important and interesting topic, an analysis of the effects of women's social mobility on fertility merits future investigations but is beyond the scope of the present study, which focuses on methodological development.

The response variable is the total number of children ever born to the respondent's wife in the OCG-1 and the total number of children ever born to the respondent in the GSS. Predictors in the analysis of educational mobility are the respondent's education level (for the OCG-1: none or elementary, 1-4 years' high school, and some college or more; for the GSS: less than high school, high school graduate, and college degree), the education level of the respondent's father, and the interaction terms between the two. For occupation, we used four categories (lower manual, upper manual, lower white-collar, and upper white-collar) to classify the father's and the respondent's occupation. See Table 5 for frequency distributions by father's and respondent's education level and occupation category.

\section{[Table 5 about here]}

As in previous research, no covariates were included in the OCG-1 analysis. Because fertility varies greatly among birth cohorts, we included cohort membership (birth cohorts born before 1945, between 1945 and 1964, and after 1965) as the sole covariate in the GSS analysis to 
facilitate comparison between the two datasets. This can be considered a fixed-effect approach to controlling for time- or cohort-related heterogeneity in fertility rates and in the estimated effects of status and mobility for the GSS data pooled over the study period.

We fit separate Poisson models using for educational and occupational mobility using the DRM and the MCM. Following Sobel (1981), we first fit three baseline models with, respectively, no mobility variables and a single salience parameter $\rho$, origin-specific $\rho$ 's, and destinationspecific $\rho$ 's. Model fit statistics (i.e., log-likelihood ratio statistics) suggested that more complicated models did not fit the data better than the simpler model with a constant $\rho$. Therefore, similar to Sobel's (1981) analysis, we fit three DRMs with single $\rho$ and one of the three mobility variables: a dummy variable indicating mobility status (nonmobile and mobile), a categorical variable indicating the direction of the social mobility (nonmobile, downwardly mobile, and upwardly mobile), and a third variable indicating the number of steps that a survey participant had traveled on the educational or occupational ladder $(0,1,2$, and 3 steps). For the MCM, we used effect coding, so the main effect can be interpreted as deviation associated with each group from the grand mean and interaction terms represent deviations from the main effects. All models were estimated using maximum likelihood.

\subsection{Results}

Because of our focus on comparing mobility effect estimates from two models, we report status effects (life-time status effects in the DRM and status main effects in the MCM) in Tables S2 and S3. Briefly, both models suggested that consistent with prior research, less education and less prestigious occupations were generally associated with higher fertility for both the OCG-1 and GSS men respondents. 
Table 6 reports the estimated salience parameter (i.e., $\hat{\rho}$; a value greater than 0.5 indicates that destination status is more salient than origin status), mobility effects (i.e., mobility status, direction, or step), and model fit statistics (deviance and the AIC statistics compared to an intercept-only model; smaller values indicate better fit to the data) in three DRMs for the OCG-1 and GSS data. For the OCG-1 data, the DRM gave negative coefficient estimates for educational mobility variables including mobility status, direction, and number of steps, but none of them were statistically or substantively significant. Only one occupational mobility variable — downward mobility—appeared to slightly reduce fertility by $10.1 \%(1-\exp (-0.107), \mathrm{p}<0.05)$. These results are consistent with those in Sobel (1981).

[Table 6 about here]

Table 7 reports mobility effect estimates-characterized as a set of origin-destination interaction contrast (i.e., $\hat{\delta}_{i j}-\hat{\delta}_{i i}$ ) -and model fit statistics from the MCM analysis of the OCG1 and GSS data; the unstructured origin-destination interaction effect estimates (i.e., $\hat{\delta}_{i j}$ ) are presented in Table S4. To illustrate, the coefficient estimate of -0.114 for the high-school-tocollege mobility group of the OCG-1 analysis in Table 7 is obtained by subtracting the interaction estimate of 0.055 for the high-school nonmobile from the interaction estimate of -0.059 for the same mobility group in Table S4. ${ }^{10}$

\section{[Table 7 about here]}

Largely consistent with previous literature, the MCM indicated that after considering the main effects of origin and destination occupation, occupational mobility appeared to be unrelated to fertility among the OCG-1 respondents. However, unlike the results using the DRM, the MCM results showed that for OCG-1 respondents, educational mobility was mostly negatively related to fertility regardless of the direction of the mobility, although not all of the mobility effect estimates 
were statistically significant. Most notably, high-school-to-college upward mobility seemed to reduce fertility by $10.8 \%(1-\exp (-0.114), \mathrm{p}<0.05)$. The college-to-high-school mobile group also had $15.5 \%(1-\exp (-0.168), \mathrm{p}<0.05)$ lower fertility due to their downward mobility from college education. The less-than-high-school-to-high-school mobility group seemed to have a slightly higher fertility rate $(\exp (0.038)-1=3.9 \%)$, but this association was not statistically significant $(\mathrm{p}>0.05)$.

The DRM analysis of the GSS data suggested that although educational mobility status was not significantly related to fertility, educational downward mobility implied $10.7 \%$ (1-exp(-0.113), $\mathrm{p}<0.05$ ) lower fertility and two-step educational mobility was associated with $12.4 \%$ (1-exp(0.132), $\mathrm{p}<0.01)$ lower fertility than the nonmobile. Neither upward nor one-step educational mobility appeared to affect the number of children per respondent. For occupational mobility, the DRM results indicated that mobility, on average, was not related to fertility. However, the models suggested that downward and upward occupational mobilities were significantly associated with fertility in opposite directions: the fertility rate of the downwardly mobile was 9.4\% (1-exp(-0.099), $\mathrm{p}<0.001)$ lower than the nonmobile, and the fertility rate of the upwardly mobile was $5.5 \%$ $(\exp (0.054)-1, \mathrm{p}<0.05)$ higher. Two-step occupational mobility also seemed to reduce fertility rates by $5.1 \%$ ( $1-\exp (-0.052), \mathrm{p}=0.055)$, but this reduction was only marginally significant.

For the GSS, the MCM found substantial educational mobility effects and some occupational mobility effects on fertility. In general, educational mobility appeared negatively associated with fertility. For example, the less-than-high-school-to-college group had 23.9\% (1$\exp (-0.273), \mathrm{p}<0.001)$ lower fertility rates that can be attributed to their upward mobility. The only exception was the high-school-to-less-than-high-school downwardly mobile group; their mobility experience was associated with $28.8 \%(\exp (0.253)-1, \mathrm{p}<0.01)$ higher fertility. For 
occupational mobility, the MCM showed that the one-step downward mobility from the parent's upper-white-collar occupation to their own lower-white-collar occupation was associated with $12.6 \%(1-\exp (-0.135), \mathrm{p}<0.01)$ fewer children than we would expect based on the main effects of the two occupation.

The deviance and the AIC statistics, where smaller values indicate a better fit, reported in Tables 6 and 7 can be used to compare the MCM and the DRM in terms of model fit. For the OCG-1 analysis, the deviance and AIC statistics are comparable between the MCM $(8,176.6$ and 19,682.7, respectively, for educational mobility; 7,970.8 and 19,477.7, respectively, for occupational mobility) and the DRM $(8,183.6$ and 19,681.9, respectively, for educational mobility; 7,980.4 and 19,469.8, respectively, for occupational mobility). ${ }^{11}$ For the GSS analysis, however, the MCM fit the data better than the DRM based on both statistics; the deviance and the AIC statistics for all MCMs are less than 7,500, whereas the deviance statistics are greater than 8,000 and the AIC greater than 22,000 for the DRMs. These results are consistent with simulation evidence and empirical findings discussed above that the MCM is more reliable than the DRM when the direction and mobility effects on fertility appeared to vary across mobility groups.

\subsection{Conclusion}

For the OCG-1 data, whereas the DRM found little support for an educational mobilityfertility association, the MCM showed substantial educational mobility effects on fertility. The difference in model results may be attributed to the mobility effect estimates shown in the upperleft panel of Table 6, which have different effect sizes and opposite directions among the offdiagonal cells so that the overall mobility effect in the DRM was diluted or concealed. For occupational mobility, the DRM implied that downward mobility reduced fertility by $10.1 \%$ (1$\exp (-0.107), \mathrm{p}<0.05)$, although none of other mobility variable estimates seemed to be consistent 
with this conclusion. The MCM did not support this conclusion in the OCG-1 data. This difference between the two models may arise from possible sources discussed earlier including different model fits and the unbalanced study design (i.e., unequal numbers of observations in each origin-destination combination) of the OCG-1 data.

The DRM and the MCM analyses of the 1974-2018 GSS data suggest qualitatively similar conclusions. However, the MCM could help researchers to pinpoint and identify mobility effects that are heterogeneous in magnitude and direction. For example, whereas the DRM results implied that occupational downward mobility reduced fertility by $9.4 \%(1-\exp (-0.099), \mathrm{p}<0.001)$, the MCM revealed that this negative relationship was primarily driven by the upper-white-collar-tolower-white-collar mobility group $(1-\exp (-0.135)=12.6 \%$ lower, $\mathrm{p}<0.01)$. For another example, the DRM found that fertility was $10.7 \%$ lower $(1-\exp (-0.113), \mathrm{p}<0.05)$ for those who experienced educational downward mobility. The MCM further showed that this overall negative association was in fact an average of the mostly negative effects of educational mobility from the lowest and highest origin statuses and the positive effect of the high-school-to-less-than-high-school mobility $(\exp (0.253)-1=28.8 \%$ higher, $\mathrm{p}<0.01)$.

Substantively, the empirical evidence from the MCM suggests that with few exceptions, intergenerational educational mobility was associated lower fertility in both the OCG-1 and the GSS data, regardless of the direction or steps of intergenerational mobility. Such results appear to be more consistent with the dissociative hypothesis (lower fertility among both the upward and downward mobility groups) than the selection hypothesis that predicts more children among the downwardly mobile and fewer children among the upwardly mobile. At the same time, the MCM analysis found that the direction of the fertility-mobility association differs depending on origin status. For example, in the GSS analysis, downward educational mobility implied $7.0 \%$ to $41.6 \%$ 
fewer children for those with college-educated parents but $28.8 \%$ more for the downward mobility group whose parents had a high-school diploma. Such heterogeneous associations merit future investigation of distinct mechanisms and responsible factors that may operate differently to link fertility with intergenerational mobility for individuals from different SES backgrounds. We caution that because of limited fertility measurement and biosocial variables, our empirical analysis is far from a full investigation of the mobility-fertility association, and the empirical evidence for examining the mobility-fertility hypothesis should be taken as suggestive not conclusive.

\section{Discussion}

In this research, we developed a new method, called the "mobility contrast model" (MCM), for investigating the heterogeneous effects of intergenerational mobility on social and demographic outcomes. Compared to the state-of-the-art diagonal reference model (DRM) - a rigorous and parsimonious model for studying mobility effects in the absence of large-scale social mobilitythe MCM provides more accurate and reliable estimates of mobility effects that differ in magnitude/size or direction, and the results are robust to the scale of intergenerational social mobile. The MCM is also flexible to include additional covariates such as age, gender, or race. Using the new method, we re-examined data from the 1962 Occupational Changes in a Generation Study (OCG-1) and the 1974 through 2018 General Social Survey (GSS) from male respondents. We found that in both data sources, educational mobility and, to a much lesser extent, occupational mobility were differentially associated with number of children depending on one's origin and/or destination status. Such heterogeneous effects of intergenerational mobility are difficult to pinpoint using the DRM. 
What model should a researcher choose to investigate intergenerational mobility effects? As illustrated in Table 8, we recommend that when the investigator has theoretical reasons to expect homogeneous mobility effects (i.e., the effect size or direction does not vary across mobility groups) and empirical evidence that intergenerational social mobility occurs at a small scale (i.e., the nonmobility group substantially outnumbers mobility groups combined), the DRM may be used as a parsimonious model that yields reliable estimates. However, when theory is lacking about the size or direction of mobility in different mobility groups, the MCM should be preferred to the DRM, especially when the data distribution suggests moderate- or large-scale intergenerational mobility. ${ }^{12}$ For researchers interested in identifying distinct mobility groups, the MCM serves such research goals better than the DRM.

\section{[Table 8 about here]}

We give four remarks to help interested readers better understand the MCM and interpret the results. First, as methodologists, our goal is to provide tools that allow researchers to describe the social processes and phenomena that they are studying. This methodological goal can be accomplished by improving algorithm and model fit, introducing a new study design (e.g., longitudinal studies compared to cross-sectional studies), or specifying new parameters (e.g., a set of interaction contrast compared to unstructured interaction terms). What ultimately matters is that the method offers insight where previous approaches do not. As we have demonstrated in the simulation study and the real-world mobility-fertility example, the MCM that we propose in the current research provides a more refined assessment of effect heterogeneity and is robust to the scale of intergenerational mobility.

Second, although we follow convention of using the term "mobility effects" in the current study, it is important to note the associational nature of our estimands. While interaction effects 
are useful for suggesting that the association between an outcome and the origin (or destination) SES depends on the destination (or origin), it requires further theoretical and methodological consideration to assess whether they represent causal effects of social mobility. The MCM should be considered a first step toward better characterizing and understanding the implication of social mobility for various social, demographic, and health outcomes.

Third, informed by the sociological concept of social mobility, the MCM quantifies mobility effects as a set of contrasts in the interaction between origin and destination main effects. In creating interaction contrasts in Step 2 of the MCM, we used origin-specific contrasts, meaning that socially mobile individuals are compared with the nonmobile of the same origin. Technically, it is possible to construct destination-specific contrasts that compare the mobile and the nonmobile of the same destination. However, as we discussed earlier, it appears that origin-specific contrasts are more closely tied to the sociological concept of social mobility than destination-specific ones. Nevertheless, one may develop many other types of interaction contrasts, but whether they are meaningful or interpretable should be guided by conceptual considerations.

Fourth, one may be concerned that modeling mobility as the interaction between the main effects of origin and destination status "prioritizes" the status effects over mobility effects. It is true that in many cases, an interaction term may be difficult to interpret besides suggesting that the effect of one variable depends on the other. In the context of mobility research, however, researchers are well positioned to interpret the interaction term in a substantively meaningful way as intergenerational mobility. Although origin and destination statuses are modeled as main effects, it does not mean that they are more important than their interaction. As we demonstrated in the fertility example, the interaction terms were substantially larger in size than the origin and 
destination main effects. To a certain degree, it is fair to say that origin, destination statuses and mobility are all abstractions.

Our empirical investigation of the mobility-fertility hypothesis focused on comparing results from the DRM and the MCM, and is thus far from a thorough treatment of the substantive topic. We note two important limitations in the empirical analysis and point to promising avenues for future research. First, to speak directly to the debate in the literature and to minimize confounding from competing factors, we followed prior research (Goldthorpe 1983; Sobel et al. 2004) and used a selected group of US-born men in the mobility-fertility analysis. To be sure, the experience of and/or the response to social mobility are likely different depending on individuals' social and demographic characteristics, so we do not assume that social mobility uniformly affects individuals' well-being (Akee et al. 2019; Breen and Whelan 1995; Cheng 2020; Cheng et al. 2019; Friedman 2014; Goldscheider and Uhlenberg 1969; Mouzon et al. 2018; Vallejo 2012). For example, women may be more susceptible to negative mobility effects because that women are more likely to be affected by interpersonal and social stressors (Matud 2004; Rudolph 2002). Family roles and relationships and their implications also differ for men and women and may mediate and/or moderate their responses to social mobility (Abbott 1987; Albright 2008; AroraJonsson 2011; Boyd 1994; Diprete and Buchmann 2006; Goldin 2006; Kravdal and Rindfuss 2008). Future research may investigate whether and how social mobility is differentially linked to fertility and other outcomes and behaviors between men and women and among sociodemographic groups.

Second, in addition to the classical OCG-1 data collected in 1962, we examined the mobility-fertility hypothesis using the more recent 1974-2018 GSS data. Because fertility decreased among younger birth cohorts, we included cohort fixed effects in the analysis to account for as much time-related heterogeneity as possible. For both the DRM and MCM analyses, an 
important assumption in this fixed-effect approach is that the effects of socioeconomic status and mobility on fertility should be similar over the study period. However, it is possible that the effects of status and social mobility on fertility and other outcomes in the 2010s may differ from those in the 1980s. Future studies may examine the validity of such an assumption by, for example, including random slopes in the MCM that allow the effects of mobility to vary among cohorts, across time periods, or over the life course. Additionally, a mixed-effects version of the MCM with random-intercepts and random-slopes can be specified to investigate time-varying variables such as employment and marital status in mobility-fertility research (see, e.g., Billingsley et al. 2018).

Lastly, the MCM can be extended to investigate questions in and beyond the field of social stratification. For example, although the methodological exposition and empirical demonstration in the present research focus on intergenerational mobility between two generations, i.e., between parents and children, the idea of the MCM can be easily extended to multigenerational studies where more than two generations are concerned. For example, two sets of interaction contrasts, one between the statuses of parents' and children's and the other between grandparents' and grandchildren's, can be simultaneously included in an MCM to estimate and infer the effects of multigenerational social mobility effects on a range of social and demographic outcomes.

The MCM approach also encourages methodological development in other areas of longstanding interest. For example, scholars of educational heterogamy are interested in how educational homogeneity or heterogeneity (i.e., marriage forms between persons of the same or different levels of educational attainment) may affect (beyond the education level of each partner) such outcomes as marriage duration, life satisfaction, and other aspects of social well-being. For another example, immigration and assimilation researchers may wish to study how age of entry, 
age at time of survey, and duration of stay (as a measure of assimilation) may affect immigrants' health and well-being. Investigators of these substantive questions have faced a methodological challenge of the same nature as in mobility effects models: The information in the third variable (e.g., educational heterogeneity; duration of stay) is completely determined by the other two (e.g., education level of each partner; age of entry and age of survey). Although the MCM is designed for studying social mobility effects, its emphasis on the correspondence between conceptual substance and statistical quantity and its focus on the interdependent and interactive effects of linearly dependent variables may be useful for addressing the aforementioned inquiries and possibly others.

\section{Notes}

${ }^{1}$ We use the terms "effect" and "affect" following the convention in this line of research without causal connotation.

${ }^{2}$ Common coding schemes or constraints include omitting one group of each variable as the reference (i.e., dummy or treatment coding) or assuming that the coefficients for each variable sum to zero (i.e., effect coding or sum-to-zero coding).

${ }^{3}$ The conundrum of the mobility model presents one of such linear dependency problems. Another well-known example of linear dependence is the age-period-cohort problem, where birth year $($ cohort $)=$ survey time (period) - age. Methodologists disagree about whether a complete and satisfactory solution exists (Fosse and Winship 2019; Luo 2013; Luo and Hodges 2020b, 2020a, 2020b; O’Brien 2014; O’Brien, Hudson, and Stockard 2008; Rodgers 1982).

${ }^{4}$ Another notable method is the "diamond model" developed by Hope (1975). However, the diamond model has serious interpretation problems about what sociological concept the model's 
parameters actually represent. That is, methodologists are not convinced that it has succeeded in establishing a correspondence between sociological substance and statistical quantity. See Sobel (1981) for a more detailed critique.

${ }^{5}$ The grand mean $\mu$ is omitted from each cell in Table 1 for clarity.

${ }^{6}$ Note that although different types of mobility variables cannot be simultaneously estimated per equation, covariates such as gender and race can be included in the DRM in a similar way as in a standard regression.

${ }^{7}$ Because the salience parameter $\rho$ is tied to origin or destination status, it is entirely possible to have different values of $\rho$ 's among mobility groups or across covariates such as gender, race, and time periods.

${ }^{8}$ For ease of illustration, the above discussion assumes two proportionality parameters for each cell; that is, each origin-destination combination has two unique $\rho$ 's. In practice, the DRM does not allow cell-specific proportionality parameters due to the limit of degrees of freedom, so as shown in Section 5's empirical example, the DRM and the MCM may have different goodness of fit to the data. Consequently, the referent based on which mobility effect is estimated in the DRM may differ from the MCM. The conceptual relationship between the DRM and the MCM, however, still holds.

${ }^{9}$ We also report results from DRMs with three origin-specific $\rho$ 's in Table S1. Although the more complex DRMs seemed to fit the data better than the simpler models with single $\rho$, the mobility effect estimates from two models are qualitatively similar. In fact, the mobility effects estimates from a DRM with a constant $\rho$ are less biased than the more complex DRMs. This is because the origin- or destination-specific $\rho$ 's represent a multiplicative cross pressure component, but whether 
these components represent mobility effects is subject to debate. See Hendrickx et al. 1993: 344348 for more detail.

${ }^{10}$ Because the unstructured interaction terms simply represent the deviations associated with each origin-destination group from the main effects of the two statuses, they may not correspond to the sociological concept of intergenerational mobility effect and thus cannot provide direct evidence about the mobility-fertility hypothesis. Nevertheless, if one were using the SAM to assess the occupational mobility-fertility relationship based on these interaction term estimates, one would reach a similar conclusion as in Blau and Duncan (1967) that little empirical evidence was found to support the occupational mobility-fertility association in the OCG-1 data.

11 The reported deviance test and AIC statistics for the DRMs are based on the best-fit DRMs among the three that we fit for each data.

${ }^{12}$ To assess the scale of intergenerational mobility in the data, interested researchers may create a frequency distribution table (see Table 5 for an example) with origin categories defining the rows and the destination the columns. The scale of intergenerational mobility can be assessed based on the ratio between the number of participants who are socially mobile and those who are socially nonmible (i.e., mobility-nonmoblity ratio). For example, we used a mobilitynonmobility ratio of 1:4 in Table 2's upper-right panel to represent a small-scale mobility scenario and a mobility-nonmobility ratio of 2:3 in Table 2's lower-right panel to generate a moderate-scale mobility scenario. 


\section{References}

Aaronson, Daniel, and Bhashkar Mazumder. 2008. "Intergenerational Economic Mobility in the United States, 1940 to 2000.” Journal of Human Resources 43(1):139-72. doi: 10.3368/jhr.43.1.139.

Abbott, Pamela. 1987. “Women's Social Class Identification: Does Husband's Occupation Make a Difference?” Sociology 21(1):91-103. doi: 10.1177/0038038587021001007.

Ahlburg, Dennis. 1998. "Intergenerational Transmission of Health." The American Economic Review 88(2):265-70.

Aiken, Leona S., Stephen G. West, and Raymond R. Reno. 1991. Multiple Regression: Testing and Interpreting Interactions. SAGE.

Akee, Randall, Maggie R. Jones, and Sonya R. Porter. 2019. "Race Matters: Income Shares, Income Inequality, and Income Mobility for All U.S. Races.” Demography. doi: 10.1007/s 13524-019-00773-7.

Albright, Karen. 2008. "In Families or as Individuals? Theoretical and Methodological Problems in the Incorporation of Women in Class Analysis." Sociology Compass 2(5):1672-89. doi: $10.1111 / \mathrm{j} .1751-9020.2008 .00156 . x$.

Arora-Jonsson, Seema. 2011. "Virtue and Vulnerability: Discourses on Women, Gender and Climate Change.” Global Environmental Change 21(2):744-51. doi: 10.1016/j.gloenvcha.2011.01.005.

Billingsley, Sunnee, Sven Drefahl, and Gebrenegus Ghilagaber. 2018. “An Application of Diagonal Reference Models and Time-Varying Covariates in Social Mobility Research on Mortality and Fertility." Social Science Research 75:73-82. doi: 10.1016/j.ssresearch.2018.06.008.

Blalock, Hubert M. 1967. "Status Inconsistency and Interaction: Some Alternative Models." American Journal of Sociology 73(3):305-15.

Blane, D., S. Harding, and M. Rosato. 1999. "Does Social Mobility Affect the Size of the Socioeconomic Mortality Differential?: Evidence from the Office for National Statistics Longitudinal Study." Journal of the Royal Statistical Society: Series A (Statistics in Society) 162(1):59-70. doi: 10.1111/1467-985X.00121.

Blau, Peter M., and Otis Dudley Duncan. 1967. "The American Occupational Structure.”

Boyd, Robert L. 1994. "Educational Mobility and the Fertility of Black and White Women." Population Research and Policy Review 13(3):275-81. doi: 10.1007/BF01074338.

Breen, Richard, and Christopher T. Whelan. 1995. "Gender and Class Mobility: Evidence from the Republic of Ireland.” Sociology 29(1):1-22. doi: 10.1177/0038038595029001002. 
Brody, Charles J., and James A. McRae. 1987. "Models for Estimating Effects of Origin, Destination, and Mobility." Social Forces 66(1):208-25. doi: 10.2307/2578908.

Chaparro, M. P., and Ilona Koupil. 2014. "The Impact of Parental Educational Trajectories on Their Adult Offspring's Overweight/Obesity Status: A Study of Three Generations of Swedish Men and Women." Social Science \& Medicine 120:199-207. doi: 10.1016/j.socscimed.2014.09.024.

Cheng, Siwei. 2020. "How to Borrow Information From Unlinked Data? A Relative Density Approach for Predicting Unobserved Distributions." Sociological Methods \& Research 0049124120926214. doi: 10.1177/0049124120926214.

Cheng, Siwei, Christopher R. Tamborini, ChangHwan Kim, and Arthur Sakamoto. 2019. "Educational Variations in Cohort Trends in the Black-White Earnings Gap Among Men: Evidence From Administrative Earnings Data.” Demography. doi: 10.1007/s13524-01900827-w.

Chetty, Raj, Nathaniel Hendren, Patrick Kline, Emmanuel Saez, and Nicholas Turner. 2014. "Is the United States Still a Land of Opportunity? Recent Trends in Intergenerational Mobility." The American Economic Review 104(5):141-47.

Chu, C. Y. Cyrus, and Hui-Wen Koo. 1990. "Intergenerational Income-Group Mobility and Differential Fertility.” The American Economic Review 80(5):1125-38.

Claussen, Bjorgulf, Jeroen Smits, Oyvind Naess, and George Davey Smith. 2005. "Intragenerational Mobility and Mortality in Oslo: Social Selection versus Social Causation." Social Science \& Medicine 61(12):2513-20. doi: 10.1016/j.socscimed.2005.04.045.

Clifford, P., and A. F. Heath. 1993. "The Political Consequences of Social Mobility." Journal of the Royal Statistical Society. Series A (Statistics in Society) 156(1):51-61. doi: $10.2307 / 2982860$.

DiPrete, Thomas A. 2002. "Life Course Risks, Mobility Regimes, and Mobility Consequences: A Comparison of Sweden, Germany, and the United States." American Journal of Sociology 108(2):267-309. doi: 10.1086/344811.

Diprete, Thomas A., and Claudia Buchmann. 2006. "Gender-Specific Trends in the Value of Education and the Emerging Gender Gap in College Completion." Demography 43(1):124. doi: 10.1353/dem.2006.0003.

Duncan, Otis Dudley. 1966. "Methodological Issues in the Analysis of Social Mobility." Pp. 5197 in Social Structureand Mobility in Economic Development. Chicago: Aldine.

Duncan, Otis Dudley. 1979. "How Destination Depends on Origin in the Occupational Mobility Table." American Journal of Sociology 84(4):793-803. 
Easterlin, Richard A. 1969. "Towards a Socioeconomic Theory of Fertility: A Survey of Recent Research on Economic Factors in American Fertility." Pp. 127-56 in Fertility and Family Planning. Ann Arbor: University of Michigan.

Easterlin, Richard A. 1976. "The Conflict between Aspirations and Resources." Population and Development Review 2(3/4):417-25. doi: 10.2307/1971619.

Fosse, Ethan, and Christopher Winship. 2019. "Analyzing Age-Period-Cohort Data: A Review and Critique." Annual Review of Sociology 45(1):467-92. doi: 10.1146/annurev-soc073018-022616.

Fox, John W. 1990. "Social Class, Mental Illness, and Social Mobility: The Social SelectionDrift Hypothesis for Serious Mental Illness." Journal of Health and Social Behavior 31(4):344-53. doi: 10.2307/2136818.

Freitas, Chanté De, Rya Buckley, Rebecca Klimo, Juliet M. Daniel, Margo Mountjoy, and Meredith Vanstone. n.d. "Admissions Experiences of Aspiring Physicians from LowIncome Backgrounds." Medical Education n/a(n/a). doi: https://doi.org/10.1111/medu.14462.

Friedman, Sam. 2014. "The Price of the Ticket: Rethinking the Experience of Social Mobility." Sociology 48(2):352-68. doi: 10.1177/0038038513490355.

Goldin, Claudia. 2006. “The Quiet Revolution That Transformed Women's Employment, Education, and Family." The American Economic Review 96(2):1-21.

Goldscheider, Calvin, and Peter R. Uhlenberg. 1969. "Minority Group Status and Fertility." American Journal of Sociology 74(4):361-72. doi: 10.1086/224662.

Goldthorpe, John H. 1983. "Women and Class Analysis: In Defence of the Conventional View." Sociology 17(4):465-88. doi: 10.1177/0038038583017004001.

Hauser, Robert M., John Robert Warren, Min-hsiung Huang, and Wendy Y. Carter. 2000. "Occupational Status, Education, and Social Mobility in the Meritocracy." in Trends in the Inheritance of Poverty and Family Structure, Page 39 Pp. 179-229 in Meritocracy and Economic Inequality. Princeton University Press.

Hendrickx, John, Nan Dirk De Graaf, Jan Lammers, and Wout Ultee. 1993. "Models for Status Inconsistency and Mobility: A Comparison of the Approaches by Hope and Sobel with the Mainstream Square Additive Model." Quality and Quantity 27(4):335-52. doi: 10.1007/BF01102497.

Hope, Keith. 1975. "Models of Status Inconsistency and Social Mobility Effects." American Sociological Review 40(3):322-43. doi: 10.2307/2094461.

Houle, Jason N. 2011. "The Psychological Impact of Intragenerational Social Class Mobility." Social Science Research 40(3):757-72. doi: 10.1016/j.ssresearch.2010.11.008. 
Houle, Jason N., and Molly A. Martin. 2011. "Does Intergenerational Mobility Shape Psychological Distress? Sorokin Revisited.” Research in Social Stratification and Mobility 29(2):193-203. doi: 10.1016/j.rssm.2010.11.001.

Hout, Michael. 1988. "More Universalism, Less Structural Mobility: The American Occupational Structure in the 1980s." American Journal of Sociology 93(6):1358-1400.

Iveson, Matthew H., and Ian J. Deary. 2017. "Intergenerational Social Mobility and Subjective Wellbeing in Later Life." Social Science \& Medicine 188:11-20. doi: 10.1016/j.socscimed.2017.06.038.

Jaccard, James, and Robert Turrisi. 2003. Interaction Effects in Multiple Regression. SAGE.

Jackson, Elton F., and Richard F. Curtis. 1972. "Effects of Vertical Mobility and Status Inconsistency: A Body of Negative Evidence." American Sociological Review 37(6):70113. doi: $10.2307 / 2093581$.

James, Sherman A., Angela Fowler-Brown, Trevillore E. Raghunathan, and John Van Hoewyk. 2006. "Life-Course Socioeconomic Position and Obesity in African American Women: The Pitt County Study." American Journal of Public Health 96(3):554-60. doi: 10.2105/AJPH.2004.053447.

Kasarda, John D., John O. G. Billy, and Kirsten West. 1986. Status Enhancement and Fertility: Reproductive Responses to Social Mobility and Educational Opportunity. Elsevier.

Kessin, Kenneth. 1971. "Social and Psychological Consequences of Intergenerational Occupational Mobility." American Journal of Sociology 77(1):1-18.

Knoke, David. 1973. "Intergenerational Occupational Mobility and the Political Party Preferences of American Men." American Journal of Sociology 78(6):1448-68. doi: $10.1086 / 225473$.

Kravdal, Øystein, and Ronald R. Rindfuss. 2008. "Changing Relationships between Education and Fertility: A Study of Women and Men Born 1940 to 1964." American Sociological Review 73(5):854-73. doi: 10.1177/000312240807300508.

Lenski, Gerhard. 1964. “COMMENT.” Public Opinion Quarterly 28(2):326-30. doi: $10.1086 / 267249$.

Lillard, Lee A., and Robert J. Willis. 1994. "Intergenerational Educational Mobility: Effects of Family and State in Malaysia." The Journal of Human Resources 29(4):1126-66. doi: $10.2307 / 146136$.

Long, Jason, and Joseph Ferrie. 2013. "Intergenerational Occupational Mobility in Great Britain and the United States Since 1850." The American Economic Review 103(4):1109-37. doi: http://dx.doi.org.ezaccess.libraries.psu.edu/10.1257/aer.103.4.1109. 
Lundberg, Olle. 1991. "Childhood Living Conditions, Health Status, and Social Mobility: A Contribution to the Health Selection Debate." European Sociological Review 7(2):149_ 62. doi: 10.1093/oxfordjournals.esr.a036593.

Luo, Liying. 2013. "Paradigm Shift in Age-Period-Cohort Analysis: A Response to Yang and Land, O'Brien, Held and Riebler, and Fienberg." Demography 50(6):1985-88. doi: 10.1007/s13524-013-0263-8.

Luo, Liying, and James S. Hodges. 2020a. "Constraints in Random Effects Age-Period-Cohort Models.” Sociological Methodology 50(1):276-317. doi: 10.1177/0081175020903348.

Luo, Liying, and James S. Hodges. 2020b. “The Age-Period-Cohort-Interaction Model for Describing and Investigating Inter-Cohort Deviations and Intra-Cohort Life-Course Dynamics:” Sociological Methods \& Research. doi: 10.1177/0049124119882451.

Mare, Robert D. 1997. "Differential Fertility, Intergenerational Educational Mobility, and Racial Inequality.” Social Science Research 26(3):263-91. doi: 10.1006/ssre.1997.0598.

Matras, Judah. 1961. "Differential Fertility, Intergenerational Occupational Mobility, and Change in the Occupational Distribution: Some Elementary Interrelationships." Population Studies 15(2):187-97. doi: 10.1080/00324728.1961.10406068.

Matud, M. Pilar. 2004. "Gender Differences in Stress and Coping Styles." Personality and Individual Differences 37(7):1401-15. doi: 10.1016/j.paid.2004.01.010.

Mouzon, Dawne M., Daphne C. Watkins, Ramona Perry, Theresa M. Simpson, and Jamie A. Mitchell. 2018. "Intergenerational Mobility and Goal-Striving Stress Among Black Americans: The Roles of Ethnicity and Nativity Status." Journal of Immigrant and Minority Health. doi: 10.1007/s10903-018-0735-4.

Nieuwbeerta, Paul, Nan Dirk de Graaf, and Wout Ultee. 2000. "The Effects of Class Mobility on Class Voting in Post-War Western Industrialized Countries.” European Sociological Review 16(4):327-48.

O'Brien, Robert. 2014. Age-Period-Cohort Models: Approaches and Analyses with Aggregate Data. CRC Press.

O’Brien, Robert M., Kenneth Hudson, and Jean Stockard. 2008. "A Mixed Model Estimation of Age, Period, and Cohort Effects." Sociological Methods \& Research 36(3):402-28. doi: 10.1177/0049124106290392.

Power, Chris, Sharon Matthews, and Orly Manor. 1996. "Inequalities in Self Rated Health in the 1958 Birth Cohort: Lifetime Social Circumstances or Social Mobility?" BMJ 313(7055):449-53. doi: 10.1136/bmj.313.7055.449.

Rodgers, Willard L. 1982. "Estimable Functions of Age, Period, and Cohort Effects." American Sociological Review 47(6):774-87. doi: 10.2307/2095213. 
Rudolph, Karen D. 2002. "Gender Differences in Emotional Responses to Interpersonal Stress during Adolescence." Journal of Adolescent Health 30(4, Suppl):3-13. doi: 10.1016/S1054-139X(01)00383-4.

Schuck, Bettina, and Nadia Steiber. 2017. "Does Intergenerational Educational Mobility Shape the Well-Being of Young Europeans? Evidence from the European Social Survey." Social Indicators Research. doi: 10.1007/s11205-017-1753-7.

Simpson, Miles E. 1970. "Social Mobility, Normlessness and Powerlessness in Two Cultural Contexts." American Sociological Review 35(6):1002-13. doi: 10.2307/2093378.

Sobel, Michael E. 1981. "Diagonal Mobility Models: A Substantively Motivated Class of Designs for the Analysis of Mobility Effects." American Sociological Review 46(6):893906. doi: $10.2307 / 2095086$.

Sobel, Michael E. 1983. "Structural Mobility, Circulation Mobility and the Analysis of Occupational Mobility: A Conceptual Mismatch.” American Sociological Review 48(5):721-27. doi: 10.2307/2094930.

Sobel, Michael E. 1985. "Social Mobility and Fertility Revisited: Some New Models for the Analysis of the Mobility Effects Hypothesis." American Sociological Review 50(5):699712. doi: $10.2307 / 2095383$.

Sobel, Michael E., Nan Dirk De Graaf, Anthony Heath, and Ying Zou. 2004. "Men Matter More: The Social Class Identity of Married British Women, 1985-1991." Journal of the Royal Statistical Society: Series A (Statistics in Society) 167(1):37-52. doi: 10.1046/j.09641998.2003.00710.x.

Song, Xi, and Robert D. Mare. 2015. "Prospective Versus Retrospective Approaches to the Study of Intergenerational Social Mobility." Sociological Methods \& Research 44(4):555-84. doi: 10.1177/0049124114554460.

Sorenson, Ann Marie. 1989. 'Husbands' and Wives' Characteristics and Fertility Decisions: A Diagonal Mobility Model.” Demography 26(1):125. doi: 10.2307/2061499.

Sorokin, Pitirim Aleksandrovich. 1927. Social Mobility. Harper \& Brothers.

Southgate, Erica, Caragh Brosnan, Heidi Lempp, Brian Kelly, Sarah Wright, Sue Outram, and Anna Bennett. 2017. "Travels in Extreme Social Mobility: How First-in-Family Students Find Their Way into and through Medical Education." Critical Studies in Education 58(2):242-60. doi: 10.1080/17508487.2016.1263223.

Stevens, Gillian. 1981. "Social Mobility and Fertility: Two Effects in One." American Sociological Review 46(5):573-85. doi: 10.2307/2094939.

Tien, H. Yuan. 1961. "The Social Mobility/Fertility Hypothesis Reconsidered: An Empirical Study." American Sociological Review 26(2):247-57. doi: 10.2307/2089862. 
Tien, H. Yuan. 1967. "Mobility, Non-Familial Activity, and Fertility." Demography 4(1):21827. doi: $10.2307 / 2060363$.

Tolsma, Jochem, Nan Dirk de Graaf, and Lincoln Quillian. 2009. "Does Intergenerational Social Mobility Affect Antagonistic Attitudes towards Ethnic Minorities?" The British Journal of Sociology 60(2):257-77. doi: 10.1111/j.1468-4446.2009.01230.x.

Torche, Florencia. 2015. "Analyses of Intergenerational Mobility: An Interdisciplinary Review." The ANNALS of the American Academy of Political and Social Science 657(1):37-62. doi: $10.1177 / 0002716214547476$.

Vallejo, Jody Agius. 2012. Barrios to Burbs: The Making of the Mexican American Middle Class. 1st ed. Stanford University Press.

Van Bavel, Jan. 2006. "The Effect of Fertility Limination on Intergenerational Social Mobility: The Quality-Quantity Trade-Off during the Demographic Tansition." Journal of Biosocial Science 38(4):553-69. doi: 10.1017/S0021932005026994.

van der Waal, Jeroen, Stijn Daenekindt, and Willem de Koster. 2017. "Statistical Challenges in Modelling the Health Consequences of Social Mobility: The Need for Diagonal Reference Models." International Journal of Public Health 62(9):1029-37. doi: 10.1007/s00038-017-1018-x.

Weakliem, David L. 1992. "Does Social Mobility Affect Political Behaviour?” European Sociological Review 8(2):153-65.

Westoff, Charles F. 1953. "The Changing Focus of Differential Fertility Research: The Social Mobility Hypothesis.” The Milbank Memorial Fund Quarterly 31(1):24-38. doi: $10.2307 / 3348422$.

Westoff, Charles F., Robert G. Potter, Philip C. Sagi, and Elliot G. Mishler. 1961. Family Growth in Metropolitan America. Princeton University Press.

Xie, Yu. 1992. "The Log-Multiplicative Layer Effect Model for Comparing Mobility Tables." American Sociological Review 57(3):380-95. doi: 10.2307/2096242.

Yaish, Meir. 2002. "The Consequences of Immigration for Social Mobility: The Experience of Israel." European Sociological Review 18(4):449-71. doi: 10.1093/esr/18.4.449.

Zang, Emma, and Nan Dirk de Graaf. 2016. "Frustrated Achievers or Satisfied Losers? Inter- and Intragenerational Social Mobility and Happiness in China." Sociological Science 3:779800. doi: $10.15195 / \mathrm{v} 3 . \mathrm{a} 33$.

Zimmer, B. G. 1981. "The Impact of Social Mobility on Fertility: A Reconsideration." Population Studies 35(1):120-31. doi: 10.1080/00324728.1981.10404956.

Zuanna, Gianpiero Dalla. 2007. "Social Mobility and Fertility." Demographic Research 17:44164. 
Table 1. Theoretical Parameters in the Square Additive Model, the Diagonal Reference Model, and the Mobility Contrast Model

\begin{tabular}{|c|c|c|c|c|c|c|c|c|c|c|}
\hline & \multirow{3}{*}{ Origin } & \multicolumn{3}{|c|}{ Square Additive Model } & \multicolumn{3}{|c|}{ Diagonal Model } & \multicolumn{3}{|c|}{ Mobility Contrast Model } \\
\hline & & & estinatio & & & Destination & & & Destinatior & \\
\hline \multirow{4}{*}{$\begin{array}{l}\text { Status } \\
\text { Effect }\end{array}$} & & 1 & 2 & 3 & 1 & 2 & 3 & 1 & 2 & 3 \\
\hline & 1 & $\alpha_{1}+\beta_{1}$ & $\alpha_{1}+\beta_{2}$ & $\alpha_{1}+\beta_{3}$ & $\theta_{1}$ & $\rho \theta_{1}+(1-\rho) \theta_{2}$ & $\rho \theta_{1}+(1-\rho) \theta_{3}$ & $\alpha_{1}+\beta_{1}$ & $\alpha_{1}+\beta_{2}$ & $\alpha_{1}+\beta_{3}$ \\
\hline & 2 & $\alpha_{2}+\beta_{1}$ & $\alpha_{2}+\beta_{2}$ & $\alpha_{2}+\beta_{3}$ & $\rho \theta_{2}+(1-\rho) \theta_{1}$ & $\theta_{2}$ & $\rho \theta_{2}+(1-\rho) \theta_{3}$ & $\alpha_{2}+\beta_{1}$ & $\alpha_{2}+\beta_{2}$ & $\alpha_{2}+\beta_{3}$ \\
\hline & 3 & $\alpha_{3}+\beta_{1}$ & $\alpha_{3}+\beta_{2}$ & $\alpha_{3}+\beta_{3}$ & $\rho \theta_{3}+(1-\rho) \theta_{1}$ & $\rho \theta_{3}+(1-\rho) \theta_{2}$ & $\theta_{3}$ & $\alpha_{3}+\beta_{1}$ & $\alpha_{3}+\beta_{2}$ & $\alpha_{3}+\beta_{3}$ \\
\hline \multicolumn{2}{|r|}{ Origin } & \multicolumn{3}{|c|}{ Destination } & \multicolumn{3}{|c|}{ Destination } & \multicolumn{3}{|c|}{ Destination } \\
\hline \multirow{4}{*}{$\begin{array}{r}\text { Mobility } \\
\text { Effect }\end{array}$} & & 1 & 2 & 3 & 1 & 2 & 3 & 1 & 2 & 3 \\
\hline & 1 & $\delta_{11}$ & $\delta_{12}$ & $\delta_{13}$ & - & $\tau_{1}$ & $\tau_{1}$ & - & $\delta_{12}-\delta_{11}$ & $\delta_{13}-\delta_{11}$ \\
\hline & 2 & $\delta_{21}$ & $\delta_{22}$ & $\delta_{23}$ & $\tau_{2}$ & - & $\tau_{1}$ & $\delta_{21}-\delta_{22}$ & - & $\delta_{23}-\delta_{22}$ \\
\hline & 3 & $\delta_{31}$ & $\delta_{32}$ & $\delta_{33}$ & $\tau_{2}$ & $\tau_{2}$ & - & $\delta_{31}-\delta_{33}$ & $\delta_{32}-\delta_{33}$ & - \\
\hline \multicolumn{2}{|c|}{$\begin{array}{c}\text { Freely-Varying } \\
\text { Parameters }\end{array}$} & & 9 & & & 7 & & & 9 & \\
\hline
\end{tabular}

Note: $\alpha_{\mathrm{i}}$ denotes the mean difference from the global mean $\mu$ (omitted in the table for presentation purpose) associated with the $i$ th origin status; $\beta_{\mathrm{j}}$ denotes the mean difference from $\mu$ associated with the $j$ th destination status; $\delta_{\mathrm{ij}}$ denotes the mean difference from origin main effects $\alpha_{\mathrm{i}}$ and destination main effects $\beta_{\mathrm{j}}$ associated with $i j$ th origin-by-destination combination in Model (2). $\theta_{\mathrm{i}}$ represents the status effect for the $i$ th life-time status on the diagonal; $\rho$ denotes a salience parameter or proportionality constraint between origin and destination status in the Diagonal Reference Model (DRM); $\tau$ denotes a mobility status parameter in the DRM. The top panel presents status effect parameters in each model. The bottom panel presents mobility effect parameters. 
Table 2. Mobility Effect and Scale Parameters, Simulated Data

\begin{tabular}{|c|c|c|c|c|c|c|}
\hline & \multicolumn{3}{|c|}{ Mobility Effect } & \multicolumn{3}{|c|}{ Mobility Scale } \\
\hline & \multicolumn{3}{|c|}{ Homogeneous } & \multicolumn{3}{|c|}{ Small } \\
\hline 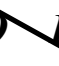 & 1 & 2 & 3 & 1 & 2 & 3 \\
\hline 1 & 0.0 & 0.4 & 0.4 & 8,000 & 1,000 & 1,000 \\
\hline 2 & 0.4 & 0.0 & 0.4 & 1,000 & 8,000 & 1,000 \\
\hline \multirow[t]{2}{*}{3} & 0.4 & 0.4 & 0.0 & 1,000 & 1,000 & 8,000 \\
\hline & \multicolumn{3}{|c|}{ Heterogeneous } & \multicolumn{3}{|c|}{ Moderate } \\
\hline & 1 & 2 & 3 & 1 & 2 & 3 \\
\hline 1 & 0.0 & -0.2 & -0.7 & 6,000 & 3,000 & 1,000 \\
\hline 2 & -0.4 & 0.0 & 1.0 & 1,000 & 6,000 & 3,000 \\
\hline 3 & 0.7 & 0.5 & 0.0 & 3,000 & 1,000 & 6,000 \\
\hline
\end{tabular}

Note: O: origin status. D: destination status. Numeric values under "Mobility Effect" are simulation parameters for each mobility group. Numbers under "Mobility Scale" are number of observations per O-D group. 
Table 3. Mobility Effect Estimates from the Diagonal Reference Model, Simulated Data

\begin{tabular}{|c|c|c|c|c|c|c|c|c|c|c|}
\hline \multirow{9}{*}{$\begin{array}{c}\text { Small-Scale } \\
\text { Mobility }\end{array}$} & & \multicolumn{4}{|c|}{ Homogeneous Mobility Effects } & \multicolumn{5}{|c|}{ Heterogeneous Mobility Effects } \\
\hline & \multirow{3}{*}{$\begin{array}{r}\rho \\
\text { Mobility } \\
\text { Down }\end{array}$} & \multirow{2}{*}{$\begin{array}{l}0.634 * * * \\
0.399 * * *\end{array}$} & \multirow[t]{2}{*}{0.593} & \multirow[t]{2}{*}{$* * *$} & \multirow[t]{2}{*}{$0.634 * * *$} & \multirow{2}{*}{$\begin{array}{l}0.611 * * * \\
0.151 * * *\end{array}$} & \multirow[t]{2}{*}{0.864} & \multirow[t]{2}{*}{$* * *$} & \multirow[t]{2}{*}{0.611} & \multirow[t]{2}{*}{$* * *$} \\
\hline & & & & & & & & & & \\
\hline & & & 0.491 & $* * *$ & & & -0.522 & $* * *$ & & \\
\hline & Up & & 0.307 & $* * *$ & & & 0.825 & $* * *$ & & \\
\hline & 1-Step & & & & $0.398 * * *$ & & & & 0.224 & $* * *$ \\
\hline & 2-Step & & & & $0.401 * * *$ & & & & 0.006 & $* * *$ \\
\hline & Deviance & 331.2 & 323.0 & & 331.2 & 2150.5 & 1748.8 & & 2090.8 & \\
\hline & AIC & -50042.3 & -50785.9 & & -50041.0 & 6083.1 & -117.9 & & 5240.4 & \\
\hline \multirow{8}{*}{$\begin{array}{c}\text { Moderate- } \\
\text { Scale } \\
\text { Mobility }\end{array}$} & $\rho$ & $0.634 * * *$ & 0.593 & $* * *$ & $0.634 * * *$ & $0.611 * * *$ & 0.864 & $* * *$ & 0.611 & $* * *$ \\
\hline & Mobility & $0.400 * * *$ & & & & $0.327 * * *$ & & & & \\
\hline & Down & & 0.525 & $* * *$ & & & -0.743 & $* * *$ & & \\
\hline & $\mathbf{U p}$ & & 0.311 & $* * *$ & & & 1.091 & $* * *$ & & \\
\hline & 1-Step & & & & $0.390 * * *$ & & & & 0.421 & $* * *$ \\
\hline & 2-Step & & & & $0.419 * * *$ & & & & 0.138 & $* * *$ \\
\hline & Deviance & 351.9 & 334.3 & & 350.3 & 3154.7 & 1926.8 & & 3009.4 & \\
\hline & AIC & -48220.7 & -49757.3 & & -48354.5 & 17579.0 & 2789.9 & & 16166.7 & \\
\hline
\end{tabular}

Note: Table figures represent mobility effects estimates from linear Diagonal Reference Models. Shaded cells indicate estimates that differ from the true parameters by 0.05 or more. For simulated data with homogeneous mobility effects, all mobility effect parameters are set to be 0.4 . For simulated data with heterogeneous mobility effects, effect parameters for mobility status, upward, downward, 1-step, and 2-step mobility are computed based on Table 2's parameters and have the following values: 0.1, $0.267,0.033,0.225$, and 0 , respectively. $\rho$ : origin-specific salience parameter; an estimate for $\rho$ larger than 0.5 indicates that destination status is more salient than origin status. AIC: Akaike information criterion. Deviance and AIC statistics are compared with an intercept-only model. $* * *: p<0.001$ 
Table 4. Mobility Effect Estimates from the Mobility Contrast Model, Simulated Data

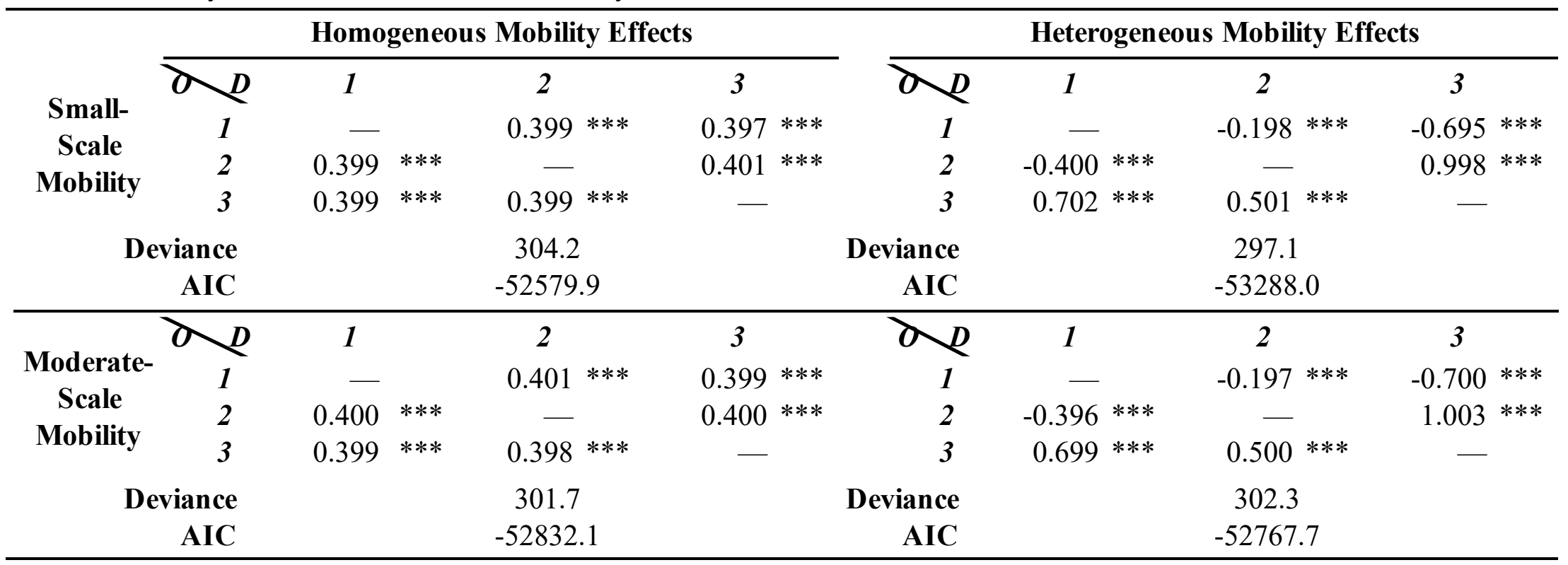

Note: Table figures represent mobility effects estimates from linear Mobility Contrast Models. See Table 2 for mobility effect parameters for generating the data. O: origin status. D: destination status. AIC: Akaike information criterion. Deviance and AIC statistics are compared with an intercept-only model. $* * *: \mathrm{p}<0.001$ 
Table 5. Frequency Distributions by Father's and Respondent's Education and Occupation, OCG-1 and GSS 1974-2018

\begin{tabular}{|c|c|c|c|c|c|c|c|c|c|}
\hline \multicolumn{5}{|c|}{ OCG-1 } & \multicolumn{5}{|c|}{ GSS } \\
\hline Father's & \multicolumn{3}{|c|}{ Respondent's Education } & & Father's & \multicolumn{3}{|c|}{ Respondent's Education } & \\
\hline Education & $<\mathbf{H S}$ & HS & Col & & Education & $<$ HS & HS & Col & \\
\hline$<$ HS & 1,481 & 1,611 & 516 & & $<$ HS & 696 & 1,714 & 530 & \\
\hline HS & 141 & 464 & 302 & & HS & 118 & 1,504 & 853 & \\
\hline Col & 29 & 127 & 255 & & Col & 13 & 291 & 664 & \\
\hline $\mathbf{N}$ & \multicolumn{3}{|c|}{4,926} & & $\mathbf{N}$ & \multicolumn{3}{|c|}{6,383} & \\
\hline Father's & \multicolumn{4}{|c|}{ Respondent's Occupation } & Father's & \multicolumn{4}{|c|}{ Respondent's Occupation } \\
\hline Occupation & $\mathbf{L M}$ & $\mathbf{U M}$ & LWC & UWC & Occupation & $\mathbf{L M}$ & $\mathbf{U M}$ & LWC & UWC \\
\hline $\mathbf{L M}$ & 1,269 & 692 & 267 & 611 & $\mathbf{L M}$ & 438 & 319 & 537 & 381 \\
\hline $\mathbf{U M}$ & 229 & 257 & 134 & 277 & $\mathbf{U M}$ & 185 & 394 & 425 & 367 \\
\hline LWC & 65 & 47 & 59 & 170 & LWC & 317 & 298 & 705 & 687 \\
\hline UWC & 117 & 109 & 114 & 476 & UWC & 153 & 110 & 320 & 790 \\
\hline $\mathbf{N}$ & \multicolumn{4}{|c|}{4,893} & $\mathbf{N}$ & \multicolumn{4}{|c|}{6,426} \\
\hline
\end{tabular}

Note: OCG-1: Occupational Changes in a Generation Study in 1962. GSS: General Social Survey. For OCG-1, <HS: none or elementary; HS: 1-4 years' high school; Col: some college or more. For GSS, $<$ HS: less than high school; HS: high school graduate; Col: college graduate. LM: lower manual; UM: upper manual; LWC: lower white-collar; UWC: upper white-collar. 
Table 6. Diagonal Reference Model Results: Educational and Occupational Mobility Effects on Fertility among US-Born White Men, OCG-1 and GSS 1974-2018

\begin{tabular}{|c|c|c|c|c|c|c|c|}
\hline & & & OCG-1 & & & GSS & \\
\hline & $\rho$ & $0.684 * * *$ & $0.700 * * *$ & $0.675 * * *$ & $0.440 * * *$ & $0.882 * * *$ & $0.259 * * *$ \\
\hline & Mobility & -0.043 & & & -0.029 & & \\
\hline Educational & Down & & -0.049 & & & $-0.113 *$ & \\
\hline Mobility & $\mathbf{U p}$ & & -0.039 & & & 0.044 & \\
\hline & 1-Step & & & -0.042 & & & -0.037 \\
\hline & 2-Step & & & -0.056 & & & $-0.132 * *$ \\
\hline Modol Fit & Deviance & 8183.7 & 8183.7 & 8183.6 & 8086.0 & 8082.3 & 8079.9 \\
\hline Mioder Fit & AIC & 19681.9 & 19683.8 & 19683.8 & 22096.1 & 22094.5 & 22092.1 \\
\hline & $\rho$ & $0.603 * * *$ & $0.819 * * *$ & $0.554 * * *$ & $0.311 * * *$ & $0.999 * * *$ & $0.220 * * *$ \\
\hline & Mobility & -0.016 & & & -0.018 & & \\
\hline Occupational & Down & & $-0.107 *$ & & & $-0.099 * * *$ & \\
\hline Mobility & Up & & 0.045 & & & $0.054 *$ & \\
\hline & 1-Step & & & 0.024 & & & 0.002 \\
\hline & 2-Step & & & -0.056 & & & -0.048 \\
\hline & 3-Step & & & -0.057 & & & -0.052 \\
\hline Medol Fit & Deviance & 7991.5 & 7980.9 & 7984.4 & 8175.4 & 8160.4 & 8171.2 \\
\hline DODUET FIt & AIC & 19478.4 & 19469.8 & 19475.3 & 22300.0 & 22284.9 & 22299.7 \\
\hline
\end{tabular}

Note: Table figures represent coefficient estimates from loglinear Diagonal Reference Models. $\rho$ : origin-specific salience parameter; an estimate for $\rho$ larger than 0.5 indicates that destination status is more salient than origin status. AIC: Akaike information criterion. Deviance and AIC statistics are compared with an intercept-only model. OCG-1: Occupational Changes in a Generation Study in 1962. GSS: General Social Survey. ***: $p<0.001 ; * *: p<0.01 ; *: p<0.05$. 
Table 7. Mobility Contrast Model Results: Educational and Occupational Mobility Effects on Fertility among US-Born White Men, OCG-1 and GSS 19742018

\begin{tabular}{|c|c|c|c|c|c|c|c|c|c|c|}
\hline \multicolumn{5}{|c|}{ OCG-1 } & \multicolumn{6}{|c|}{ GSS } \\
\hline \multirow[t]{2}{*}{ Origin } & \multicolumn{3}{|c|}{ Education Destination } & & Origin & \multicolumn{4}{|c|}{ Education Destination } & \\
\hline & $<\mathbf{H S}$ & HS & Col & & & $<$ HS & & HS & Col & \\
\hline$<\mathbf{H S}$ & - & 0.038 & -0.016 & & $<\mathbf{H S}$ & - & & $-0.211 * *$ & $-0.273 * * *$ & \\
\hline HS & -0.051 & - & $-0.114 *$ & & HS & 0.253 & $* *$ & - & -0.011 & \\
\hline Col & -0.078 & $-0.168 *$ & - & & Col & -0.537 & $* * *$ & -0.073 & - & \\
\hline Deviance & & & & & Deviance & & & 7328.0 & & \\
\hline $\mathbf{A I C}$ & & & & & AIC & & & 7348.5 & & \\
\hline \multirow[t]{2}{*}{ Origin } & \multicolumn{4}{|c|}{ Occupation Destination } & Origin & \multicolumn{5}{|c|}{ Occupation Destination } \\
\hline & $\mathbf{L M}$ & $\mathbf{U M}$ & LWC & UWC & & $\mathbf{L M}$ & & $\mathbf{U M}$ & LWC & $\mathbf{U W C}$ \\
\hline $\mathbf{L M}$ & - & -0.019 & -0.093 & -0.080 & $\mathbf{L M}$ & - & & 0.043 & 0.010 & -0.024 \\
\hline $\mathbf{U M}$ & -0.012 & - & 0.151 & 0.004 & $\mathbf{U M}$ & 0.003 & & - & 0.055 & -0.019 \\
\hline LWC & 0.085 & 0.127 & - & 0.105 & LWC & -0.078 & & -0.038 & - & -0.027 \\
\hline $\mathbf{U W C}$ & -0.044 & -0.079 & -0.029 & - & $\mathbf{U W C}$ & 0.005 & & -0.074 & $-0.135 * *$ & - \\
\hline Deviance & \multicolumn{4}{|c|}{7970.8} & Deviance & \multicolumn{5}{|c|}{7411.3} \\
\hline AIC & \multicolumn{4}{|c|}{19477.7} & AIC & \multicolumn{5}{|c|}{7448.3} \\
\hline
\end{tabular}

Note: Table figures represent mobility contrast estimates from loglinear Mobility Contrast Models. AIC: Akaike information criterion. Deviance and AIC statistics are compared with an intercept-only model. OCG-1: Occupational Changes in a Generation Study in 1962. GSS: General Social Survey. For OCG-1, $<$ HS: none or elementary; HS: 1-4 years' high school; Col: some college or more. For GSS, <HS: less than high school; HS: high school graduate; Col: college graduate. LM: lower manual; UM: upper manual; LWC: lower white-collar; UWC: upper white-collar. ${ }^{* * *}$ : $\mathrm{p}<0.001 ; * *: \mathrm{p}<0.01 ; *: \mathrm{p}<0.05$. 
Table 8. Diagonal Reference Model or Mobility Contrast

Model: Best-Practice Recommendations

\begin{tabular}{lcc}
\hline Mobility Effect & \multicolumn{2}{c}{ Mobility Scale } \\
\cline { 2 - 3 } Homogeneous & Small & Moderate / Large \\
Heterogeneous & DRM & DRM or MCM \\
\hline
\end{tabular}

Note: DRM: the diagonal referece model; MCM: the mobility contrast model. 
Table S1. Mobility Effect Estimates from the Diagonal Reference Model with Multiple Salience Parameters, Simulated Data

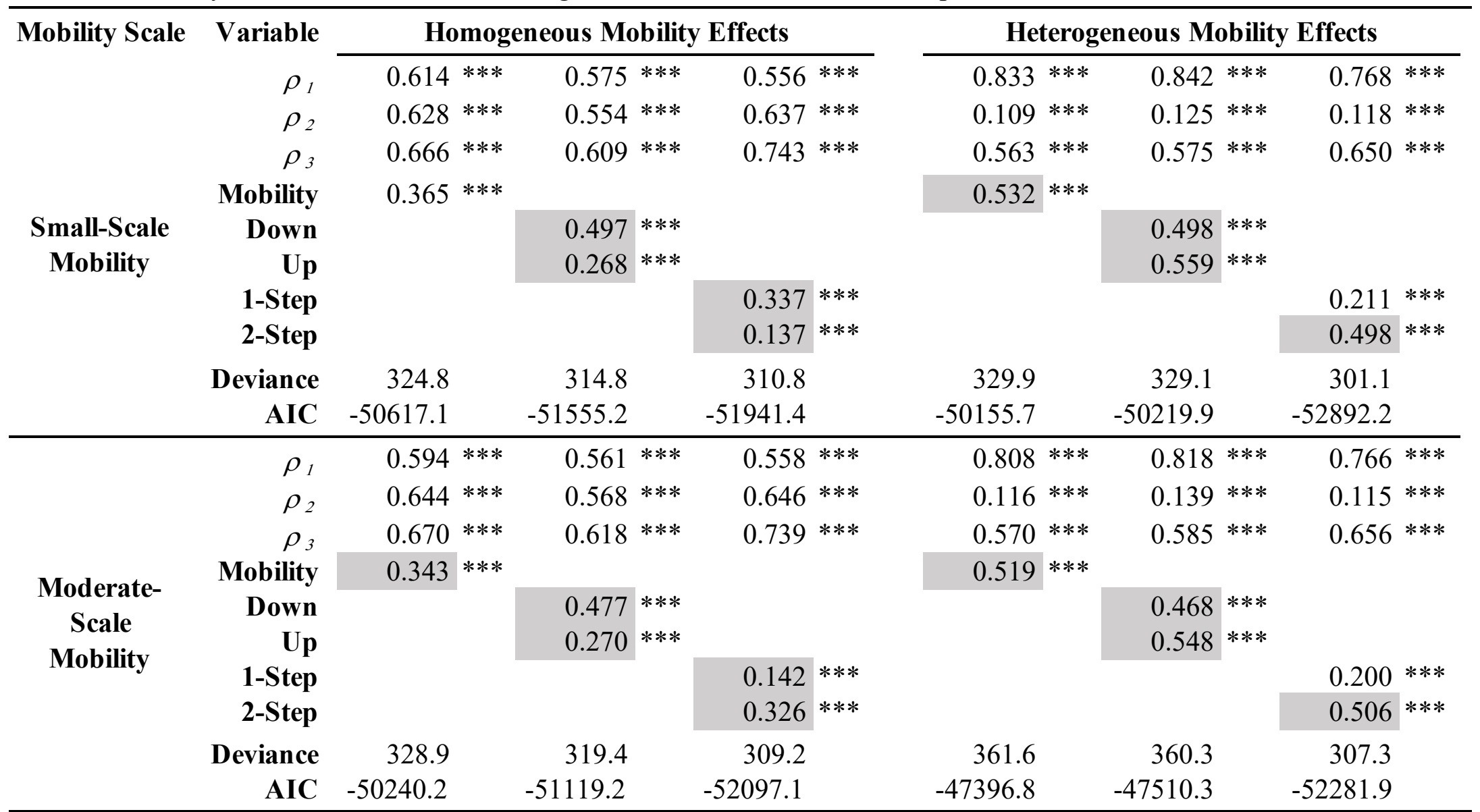

Note: Table figures represent mobility effects estimates from linear Diagonal Reference Models. Shaded cells indicate estimates that differ from the true parameters by 0.05 or more. For simulated data with homogeneous mobility effects, all mobility effect parameters are set to be 0.4 . For simulated data with heterogeneous mobility effects, effect parameters for mobility status, upward, downward, 1-step, and 2-step mobility are computed based on Table 2's parameters and have the following values: 0.1, $0.267,0.033,0.225$, and 0 , respectively. $\rho$ : origin-specific salience parameter; an estimate for $\rho$ larger than 0.5 indicates that destination status is more salient than origin status. AIC: Akaike information criterion. Deviance and AIC statistics are compared with an intercept-only model. ***: $\mathrm{p}<0.001$ 


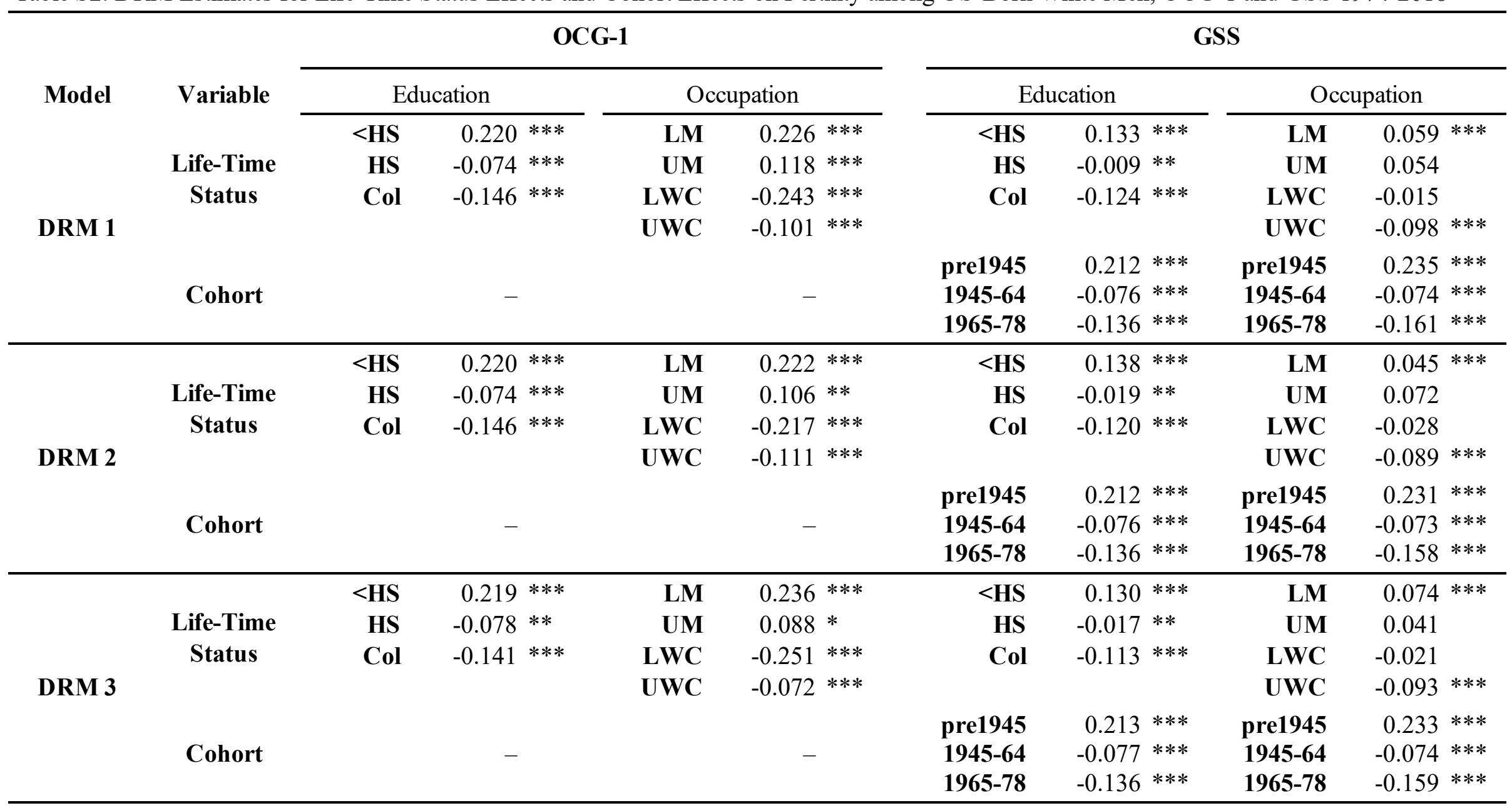

Note: Table figures represent main effect estimates from loglinear Diagonal Reference Models using effect coding so that the effects of a variable add up to zero. DRM: diagonal reference model. DRM 1: DRM with a mobility status dummy variable. DRM 2: DRM with a mobility direction categorical variable. DRM 3: DRM with a mobility step categorical variable. OCG-1: Occupational Changes in a Generation Study in 1962. GSS: General Social Survey. For OCG-1, $<$ HS: none or elementary; HS: 1-4 years' high school; Col: some college or more. For GSS, $<$ HS: less than high school; HS: high school graduate; Col: college graduate. LM: lower manual; UM: upper manual; LWC: lower white-collar; UWC: upper white-collar. $* * *: \mathrm{p}<0.001 ; * *: \mathrm{p}<0.01 ; *: \mathrm{p}<0.05$. 


\begin{tabular}{|c|c|c|c|c|c|c|c|c|c|c|c|}
\hline \multirow[b]{2}{*}{ Model } & \multirow{3}{*}{$\begin{array}{l}\text { Variable } \\
\text { Intercept }\end{array}$} & \multicolumn{5}{|c|}{ OCG-1 } & \multicolumn{5}{|c|}{ GSS } \\
\hline & & \multicolumn{3}{|c|}{ Education } & \multicolumn{2}{|c|}{ Occupation } & \multicolumn{3}{|c|}{ Education } & \multicolumn{2}{|c|}{ Occupation } \\
\hline \multirow{12}{*}{$\begin{array}{c}\text { Mobility } \\
\text { Contrast } \\
\text { Model }\end{array}$} & & & 0.805 & $* * *$ & & $0.778 * * *$ & & 0.643 & $* * *$ & & $0.718 * * *$ \\
\hline & & $<\mathbf{H S}$ & 0.066 & $* *$ & LM & $0.094 * * *$ & $<$ HS & 0.131 & $* * *$ & LM & $0.051 * * *$ \\
\hline & Orixin & HS & -0.025 & & UM & 0.031 & HS & 0.099 & $* *$ & $\mathbf{U M}$ & 0.016 \\
\hline & Origmil & Col & -0.042 & & LWC & -0.064 & Col & -0.230 & $* * *$ & LWC & -0.008 \\
\hline & & & & & UWC & -0.061 & & & & UWC & $-0.059 *$ \\
\hline & & $<$ HS & 0.183 & $* * *$ & LM & $0.110 * * *$ & $<\mathbf{H S}$ & -0.078 & & LM & -0.004 \\
\hline & D ti. & HS & -0.084 & $* *$ & UM & $0.089 * *$ & HS & 0.048 & & UM & $0.045 *$ \\
\hline & Destination & Col & -0.099 & $* * *$ & LWC & $-0.158 * * *$ & Col & 0.031 & & LWC & -0.014 \\
\hline & & & & & UWC & -0.041 & & & & UWC & -0.027 \\
\hline & & & & & & & pre1945 & 0.205 & $* * *$ & pre1945 & $0.220 * * *$ \\
\hline & Cohort & & - & & & - & $1945-64$ & -0.057 & $* * *$ & 1945-64 & $-0.056 * * *$ \\
\hline & & & & & & & $1965-78$ & -0.147 & $* * *$ & $1965-78$ & $-0.164 * * *$ \\
\hline
\end{tabular}

Note: Table figures represent main effect estimates from loglinear Mobility Contrast Models using effect coding so that the effects of a variable add up to zero. MCM: Mobility Contrast Model. OCG-1: Occupational Changes in a Generation Study in 1962. GSS: General Social Survey. For OCG-1, $<$ HS: none or elementary; HS: 1-4 years' high school; Col: some college or more. For GSS, <HS: less than high school; HS: high school graduate; Col: college graduate. LM: lower manual; UM: upper manual; LWC: lower white-collar; UWC: upper white-collar. ${ }^{* * *}: \mathrm{p}<0.001 ;{ }^{* *}$ : $<<0.01 ;{ }^{*}$ : $\mathrm{p}<0.05$. 
Table S4. Square Additive Model Results (Estimated Interactions in Step 1 of the MCM): Educational and Occupational Mobility Effects on Fertility among US-Born White Men, OCG-1 and GSS 1974-2018

\begin{tabular}{|c|c|c|c|c|c|c|c|c|c|}
\hline \multicolumn{5}{|c|}{ OCG-1 } & \multicolumn{5}{|c|}{ GSS } \\
\hline \multirow[t]{2}{*}{ Origin } & \multicolumn{3}{|c|}{ Education Destination } & & \multirow[t]{2}{*}{ Origin } & \multicolumn{3}{|c|}{ Education Destination } & \\
\hline & $<\mathbf{H S}$ & HS & Col & & & $<$ HS & HS & Col & \\
\hline$<\mathbf{H S}$ & -0.008 & 0.031 & -0.023 & & $<\mathbf{H S}$ & $0.162 * *$ & -0.050 & $-0.112 * * *$ & \\
\hline HS & 0.004 & 0.055 & -0.059 & & HS & $0.172 * *$ & $-0.080 *$ & $-0.092 * *$ & \\
\hline Col & 0.004 & -0.086 & 0.082 & & Col & $-0.334 * *$ & $0.130 *$ & $0.204 * * *$ & \\
\hline \multirow[t]{2}{*}{ Origin } & \multicolumn{4}{|c|}{ Occupation Destination } & Origin & \multicolumn{3}{|c|}{ Occupation Destination } & \\
\hline & $\mathbf{L M}$ & $\mathbf{U M}$ & LWC & UWC & & $\mathbf{L M}$ & $\mathbf{U M}$ & LWC & UWC \\
\hline $\mathbf{L M}$ & 0.048 & 0.029 & -0.045 & -0.032 & $\mathbf{L M}$ & -0.007 & 0.036 & 0.003 & -0.031 \\
\hline $\mathbf{U M}$ & -0.048 & -0.036 & $0.115 *$ & -0.032 & $\mathbf{U M}$ & -0.007 & -0.010 & 0.045 & -0.029 \\
\hline LWC & 0.005 & 0.048 & -0.079 & 0.026 & LWC & -0.042 & -0.002 & 0.036 & 0.009 \\
\hline UWC & -0.006 & -0.041 & 0.009 & 0.038 & UWC & 0.056 & -0.023 & $-0.084 *$ & 0.051 \\
\hline
\end{tabular}

Note: Table figures represent origin-destination interaction estimates from loglinear Square Additive Models using effect coding so that the interaction estimates in each row and each column sum to zero. These interaction effect estimates are identical to Step 1 of the Mobility Contrast Model. Interaction effects represent the deviation in each origin-destination cell from the expectation based on their origin and destination main effects. OCG-1: Occupational Changes in a Generation Study in 1962. GSS: General Social Survey. For OCG-1, <HS: none or elementary; HS: 1-4 years' high school; Col: some college or more. For GSS, <HS: less than high school; HS: high school graduate; Col: college graduate. LM: lower manual; UM: upper manual; LWC: lower white-collar; UWC: upper white-collar. ${ }^{* * *}$ : $\mathrm{p}<0.001 ; * *$ : $<0.01 ; *$ : $<<0.05$. 